\title{
Linguistic hesitant intuitionistic fuzzy cross-entropy measures
}

\author{
Wei Yang* Yongfeng Pang Jiarong Shi \\ Department of Mathematics, School of Science, \\ Xi'an University of Architecture and Technology, 710055 Xi'an, Shaanxi, P. R. China
}

Received 19 November 2015

Accepted 10 September 2016

\begin{abstract}
In this paper, several cross-entropy measures for linguistic hesitant intuitionistic fuzzy information have been developed which integrating cross-entropy measures of intuitionistic fuzzy sets and hesitant fuzzy linguistic sets. Some desirable properties of new cross-entropy measures have been studied. Two new multiple attribute decision making methods have been presented based on the new cross-entropy measures in which attribute values are given in the form of linguistic hesitant intuitionistic fuzzy values to reflect human hesitantation and fuzzy thinking comprehensively. We consider different attribute weight situations including attribute weights are completely known, partly known and completely unknown. An optimization model is established to determine attribute weights if they are partly known and a formula is given if attribute weights are completely unknown. Finally, a numerical example is presented to illustrate practical advantages and effectiveness of the proposed approaches.
\end{abstract}

Keywords: Hesitant fuzzy set; intuitionisic fuzzy set; linguistic argument; aggregation operator; linguistic hesitant intuitionistic fuzzy cross-entropy.

\section{Introduction}

Fuzziness and uncertainty exists extensively in decision making process due to complicated decision problems, limited decision time and fuzzy nature of human thinking, etc. Many useful tools have been developed including fuzzy set, intuitionistic fuzzy set $^{1}$, hesitant fuzzy set ${ }^{2-3}$, linguistic arguments ${ }^{4-8}$, etc. In fuzzy set, the membership of each element is a real number between 0 and 1 . Intuitionistic fuzzy set is the extension of fuzzy set, which is characterized by membership and non-membership summing less than 1. Hesitant fuzzy set is another extension of fuzzy set, in which several values are possible for the definition of a membership function of a fuzzy set. The envelope of hesitant fuzzy set is intuitionistic fuzzy set. Compar- ing with other tools to model fuzzy and uncertain information, hesitant fuzzy set is more powerful and accurate. The hesitant fuzzy set has been studied and applied extensively ${ }^{9-14}$. Some hesitant aggregation operators have been proposed ${ }^{15-17}$. Some classic multiple attribute decision making methods have been extended to hesitant fuzzy environment ${ }^{18-20}$. Some distance measures, entropy measures and correlation coefficients have been generalized to accommodate hesitant fuzzy information ${ }^{21-25}$. Hesitant fuzzy set has been extended to accommodate intuitionistic fuzzy values ${ }^{26}$, interval-values ${ }^{27}$, triangular fuzzy values ${ }^{28}$, linguistic arguments ${ }^{29-35}$, etc. Due to fuzzy nature of human thinking, complicated decision problems and limited decision time, decision makers would like to evaluate with linguistic terms rather than exact numerical values. Sev-

\footnotetext{
* Corresponding author. E-mail: yangweipyf@163.com.
} 
eral types of linguistic models have been developed. Herrera and Martínez ${ }^{4}$ proposed 2-tuple linguistic model to avoid information distortion and loss. Dong et al. ${ }^{5}$ defined numerical scale and extended the 2-tuple fuzzy linguistic representation models under numerical scale. By using 2-tuple linguistic model, each evaluation value only has one linguistic evaluation value. Rodríguez et al. ${ }^{32}$ developed hesitant linguistic fuzzy set in which each element has several linguistic terms. Wang ${ }^{35}$ generalized hesitant fuzzy linguistic term sets by enabling any non-consecutive linguistic terms in them. Pang et al. ${ }^{6}$ developed probabilistic linguistic term set in which possible linguistic values may have different importance degrees. The fuzzy linguistic modelling based on discrete fuzzy numbers is proposed by Riera et al. ${ }^{7}$ to manage hesitant fuzzy linguistic information. Comparing with fuzzy numbers, intuitionistic fuzzy values are more accurately to model hesitation. The concept of a possibility distribution for hesitant fuzzy linguistic information has been defined ${ }^{11}$. But hesitation in decision making hasn't been modeled properly by existing linguistic models. In decision making process, experts would express some hesitation in evaluating with linguistic terms. Intuitionistic fuzzy values can be used to model hesitation accurately. By using intuitionistic fuzzy values to model hesitation in linguistic evaluating process, fuzzy nature of human thinking can be reflected accurately and hesitation can be modeled properly. If an expert uses linguistic term $s_{\alpha}$ in evaluating some alternative with respect to some attribute and he/she thinks the membership of alternative satisfying the attribute is $\mu$ and nonmembership is $v$, then linguistic intuitionistic fuzzy element (LIFE) $\left(s_{\alpha},(\mu, v)\right)$ can be got. If two experts use the same linguistic term and different intuitionistic fuzzy values, intuitionistic fuzzy values are merged together. For example, in evaluating performance of a candidate for a college dean, one expert thinks the degree of 'slightly good $\left(S_{6}\right)$ ' the candidate belonging to is $(0.6,0.3)$ and degree of ' $g o o d$ $\left(S_{7}\right)^{\prime}$ is $(0.7,0.2)$. Another expert thinks the degree of 'fair $\left(S_{5}\right)$ ' the candidate belonging to is $(0.5,0.4)$, the degree of 'slightly good' is $(0.7,0.1)$ and the degree of 'good' is $(0.6,0.2)$. Then we can get lin- guistic hesitant intuitionistic fuzzy information as $\breve{h}=\left\{\left(S_{5},(0.5,0.4)\right),\left(S_{6},(0.6,0.3),(0.7,0.1)\right),\left(S_{7}\right.\right.$, $(0.6,0.2),(0.7,0.2))\}$. The linguistic hesitant intuitionistic fuzzy set is developed by Yang et al. ${ }^{36}$. Comparing with other tools, linguistic hesitant intuitionisic fuzzy values can model fuzzy and uncertain information existing in decision making process more accurate, which is the prerequisite to get scientific and reasonable decision-making results.

A growing number of studies focus on entropy measures and cross-entropy measures due to advantages of measuring fuzziness and discrimination information ${ }^{37-49}$. Kullback and Leibler ${ }^{39}$ defined a cross-entropy measure between two probability distribution. The fuzzy cross-entropy has been defined by Bhandari and $\mathrm{Pal}^{40}$ by using its membership function. Zhang and Jiang ${ }^{41}$ developed vague crossentropy by analogy with the cross-entropy of probability distributions. Chen et al. ${ }^{42}$ developed several cross-entropy measures for uncertain variables. Mao et al. ${ }^{43}$ proposed a novel symmetric intuitionistic fuzzy cross-entropy formula taking into account intuitionistic fuzzy entropy and fuzzy entropy simultaneously. Xia and $\mathrm{Xu}^{44}$ defined two cross-entropy measures for intuitionistic fuzzy values by normalizing the J-divergence intuitionistic fuzzy values introduced by Hung and Yang ${ }^{45}$. Wang and $\mathrm{Li}^{46}$ proposed a cross-entropy measure of the membership degree from the non-membership degree for intuitionistic fuzzy values. Qi et al. ${ }^{47}$ defined a new generalized interval-valued intuitionistic fuzzy crossentropy measure and gave a new method to determine unknown attribute weights and expert weights based on the new cross-entropy measure. $\mathrm{Xu}$ and $\mathrm{Xia}^{48}$ defined two cross-entropy measures for hesitant fuzzy information. Peng et al. ${ }^{26}$ have developed some fuzzy cross-entropy measures for intuitionistic hesitant fuzzy information. The cross-entropy methods have been used extensively, such as traffic signal optimization, portfolio selection, clustering, energy management, etc.

From the above analysis we can find that all the existing cross-entropy measures are based on exact numerical values, fuzzy values, intuitionistic fuzzy values, hesitant fuzzy values. Linguistic hesitant intuitionistic fuzzy values are more accurate and flex- 
ible in modeling fuzzy and uncertain information. However, the study on cross-entropy measures for linguistic hesitant intuitionistic fuzzy information hasn't been found yet. Due to the fact hesitation is common existing in actual decision making process, it is necessary to develop some cross entropy measures for linguistic hesitant intuitionistic fuzzy information. The aim of this paper is to propose several linguistic hesitant intuitionistic fuzzy cross-entropy measures by extending intuitionistic fuzzy crossentropy measures and hesitant fuzzy cross-entropy measures to linguistic hesitant intuitionistic fuzzy environment, which can comprehensively accommodate membership, nonmembership and hesitation degree in linguistic evaluation process. Based on the new cross-entropy measures, a programming model is set up to derive unknown attribute weights by considering deviation between attribute assessment values and a formula is given to derive attribute weights if they are completely unknown. Then we develop two algorithms based on the new cross-entropy measures integrating the afore presented methods considering different situations of attribute weights. A numerical example of supplier selection problem is presented to illustrate the new algorithms. Additionally, it is important to note that the decision making methods proposed in this paper can also be used to solve other decision making problems with high uncertainty and hesitation degrees.

In order to do so, the rest of the paper is organized as follows. In section 2, we first review some basic concepts on linguistic hesitant intuitionistic fuzzy set. Then we define several linguistic hesitant intuitionistic fuzzy aggregation operators. In section 3, several cross-entropy measures for linguistic hesitant intuitionistic fuzzy information have been developed and some desirable properties have been studied. In section 4 , we propose two new multiple attribute decision making methods based on the new cross-entropy measures. In section 5, an example of supplier selection is given to illustrate feasibility and practical advantages of new methods. The conclusions are given in the last section.

\section{Linguistic hesitant intuitionistic fuzzy term set}

An HFS is defined in terms of a function that returns a set of membership values of each element in the domain.

Definition 2.1 ${ }^{2}$. Let $X$ be a reference set, an HFS $A$ on $X$ is a function $h$ that returns a subset of values in $[0,1]$ when it is applied to $X$ :

$$
A=\left\{<x, h_{A}(x)>\mid x \in X\right\},
$$

where $h_{A}(x)$ is a set of some different values in $[0,1]$, representing the possible membership degrees of $x \in X$ to $A$. $h_{A}(x)$ is called a hesitant fuzzy element (HFE) ${ }^{14}$.

Suppose that $S=\left\{s_{i} \mid i=1, \ldots, g\right\}$ is a finite and totally ordered discrete term set, where $s_{i}$ represents a possible value for a linguistic variable. A set of nine terms $S^{50}$ can be expressed as follows $S=\left\{s_{1}=\right.$ extremely poor, $s_{2}=$ very poor, $s_{3}=$ poor, $s_{4}=$ slightly poor, $s_{5}=$ fair, $s_{6}=$ slightly good, $s_{7}=$ good, $s_{8}=$ very good, $s_{9}=$ extremely good $\}$. In order to preserve all information, the discrete linguistic term set $S$ can be extended to a continuous one $\bar{S}=\left\{s_{\alpha} \mid s_{0} \leqslant s_{\alpha} \leqslant s_{g}, \alpha \in[0, g]\right\}$.

By extending hesitant fuzzy set, Zhang and $\mathrm{Wu}^{31}$ develop hesitant fuzzy linguistic term set (HFLS), in which a linguistic variable has several linguistic terms.

Definition 2.2. Let $X$ be a reference set and $\bar{S}=\left\{s_{\alpha} \mid s_{0} \leqslant s_{\alpha} \leqslant s_{g}\right\}$ be a linguistic term set. A hesitant fuzzy linguistic term set $\bar{A}$ on $X$ is an ordered finite subset of the consecutive linguistic term set $\bar{S}$

$$
\bar{A}=\left\{<x_{i}, \bar{h}_{\bar{A}}\left(x_{i}\right)>\mid x_{i} \in X, i=1,2, \ldots, n\right\},
$$

where $\bar{h}_{\bar{A}}\left(x_{i}\right): X \rightarrow \bar{S}$ denotes all the possible linguistic evaluation values of element $x_{i} \in X$. For convenience, we call $\bar{h}_{\bar{A}}\left(x_{i}\right)$ a hesitant fuzzy linguistic element (HFLE), which can be represented as

$$
\bar{h}_{\bar{A}}\left(x_{i}\right)=\left\{s_{i} \mid s_{i} \in \bar{h}_{\bar{A}}\left(x_{i}\right)\right\},
$$

here $s_{i}$ is a linguistic argument.

Since experts would express some hesitation in evaluation using linguistic terms, we use intuitionistic fuzzy value to model hesitation. If multiple experts evaluate alternatives with respect to attributes 
using different linguistic terms and different intuitionistic fuzzy values, we get linguistic hesitant intuitionistic fuzzy set, which can be defined as follows.

Definition $2.3^{36}$. Let $X=\left\{x_{1}, x_{2}, \ldots, x_{n}\right\}$ be a reference set and $\bar{S}=\left\{s_{\alpha} \mid s_{0} \leqslant s_{\alpha} \leqslant s_{g}\right\}$ be a linguistic term set. A linguistic hesitant intuitionistic fuzzy set (LHIFS) $\check{A}$ on $X$ is defined as

$$
\check{A}=\left\{<x_{i}, \breve{h}_{\breve{A}}\left(x_{i}\right)>\mid x_{i} \in X, i=1,2, \ldots, n\right\},
$$

where $\breve{h}_{\breve{A}}\left(x_{i}\right): X \rightarrow H$ denotes all possible linguistic intuitionistic fuzzy evaluation values of element $x_{i} \in X$. For convenience, we call $\breve{h}_{\breve{A}}\left(x_{i}\right)$ a linguistic hesitant intuitionistic fuzzy element (LHIFE), which can be represented as

$$
\breve{h}_{\breve{A}}\left(x_{i}\right)=\left\{\left(s_{\theta_{i}}, \operatorname{lh}\left(s_{\theta_{i}}\right)\right) \mid x_{i} \in X\right\},
$$

$s_{\theta_{i}}$ is a linguistic argument and $\operatorname{lh}\left(s_{\theta_{i}}\right)=$ $\left\{\left(\mu_{i}^{(k)}, v_{i}^{(k)}\right)\right\}$ is the set of intuitionistic fuzzy membership values that $s_{\theta_{i}}$ satisfies $x_{i} .\left(s_{\theta_{i}}, \operatorname{lh}\left(s_{\theta_{i}}\right)\right)$ is the linguistic intuitionistic fuzzy element (LIFE). Let $H$ be the set of all LHIFEs.

Definition $\mathbf{2 . 4}^{36}$. Let $\breve{h}, \breve{h}_{1}$ and $\breve{h}_{2}$ be LHIFEs, $\lambda>0 . a_{k}=\left(s_{\theta_{k}}, \operatorname{lh}\left(s_{\theta_{k}}\right)\right) \in \breve{h}, a_{i}=\left(s_{\theta_{i}}, \operatorname{lh}\left(s_{\theta_{i}}\right)\right) \in \breve{h}_{1}$, $a_{j}=\left(s_{\theta_{j}}, \operatorname{lh}\left(s_{\theta_{j}}\right)\right) \in \breve{h}_{2}$. Some operations on these LHIFEs can be defined as follows

(1) $\breve{h}_{1} \oplus \breve{h}_{2}=\bigcup_{a_{i} \in \breve{h}_{1}, a_{i} \in \check{h}_{2}} \quad\left\{\left(s_{\theta_{i}+\theta_{j}}\right.\right.$, $\bigcup_{\left(\mu_{i}^{(l)}, v_{i}^{(l)}\right) \in \operatorname{lh}\left(s_{\theta_{i}}\right),\left(\mu_{j}^{(m)}, v_{j}^{(m)}\right) \in \operatorname{lh}\left(s_{\theta_{j}}\right)}\left\{\left(\mu_{i}^{(l)}+\mu_{j}^{(m)}-\right.\right.$ $\left.\left.\left.\left.\mu_{i}^{(l)} \mu_{j}^{(m)}, v_{i}^{(l)} v_{j}^{(m)}\right)\right\}\right)\right\}$,

(2) $\check{h}_{1} \otimes \check{h}_{2}=\bigcup_{a_{i} \in \breve{h}_{1}, a_{j} \in \check{h}_{2}}\left\{\left(s_{\theta_{i} \theta_{j}}\right.\right.$, $\bigcup_{\left(\mu_{i}^{(l)}, v_{i}^{(l)}\right) \in \operatorname{lh}\left(s_{\theta_{i}}\right),\left(\mu_{j}^{(m)}, v_{j}^{(m)}\right) \in \operatorname{lh}\left(s_{\theta_{j}}\right)} \quad\left\{\left(\mu_{i}^{(l)} \mu_{j}^{(m)}, v_{i}^{(l)}+\right.\right.$ $\left.\left.\left.\left.v_{j}^{(m)}-v_{i}^{(l)} v_{j}^{(m)}\right)\right\}\right)\right\}$,

(3) $\lambda \breve{h}=\bigcup_{a_{k} \in \check{h}}\left\{\left(s_{\lambda \theta_{k}}, \bigcup_{\left(\mu_{k}^{(n)}, v_{k}^{(n)}\right) \in \operatorname{lh}\left(s_{\theta_{k}}\right)}\{(1-\right.\right.$ $\left.\left.\left.\left.\left(1-\mu_{k}^{(n)}\right)^{\lambda},\left(v_{k}^{(n)}\right)^{\lambda}\right)\right\}\right)\right\}$,

(4) $(\breve{h})^{\lambda}=\bigcup_{a_{k} \in \check{h}}\left\{\left(s_{\theta_{k}^{\lambda}}, \bigcup_{\left(\mu_{k}^{(n)}, v_{k}^{(n)}\right) \in \operatorname{lh}\left(s_{\theta_{k}}\right)}\left\{\left(\left(\mu_{k}^{(n)}\right)^{\lambda}\right.\right.\right.\right.$, $\left.\left.\left.\left.1-\left(1-v_{k}^{(n)}\right)^{\lambda}\right)\right\}\right)\right\}$.

Theorem $1^{36}$. Let $\breve{h}, \breve{h}_{1}$ and $\breve{h}_{2}$ be LHIFEs and $\lambda, \lambda_{1}, \lambda_{2}>0$, then

(1) $\breve{h}_{1} \oplus \breve{h}_{2}=\breve{h}_{2} \oplus \breve{h}_{1}$,

(2) $\breve{h}_{1} \otimes \check{h}_{2}=\check{h}_{2} \otimes \check{h}_{1}$,

(3) $\lambda\left(\check{h}_{1} \oplus \check{h}_{2}\right)=\lambda \breve{h}_{1} \oplus \lambda \check{h}_{2}$,
(4) $\left(\check{h}_{1} \otimes \check{h}_{2}\right)^{\lambda}=\left(\check{h}_{1}\right)^{\lambda} \otimes\left(\check{h}_{2}\right)^{\lambda}$,

(5) $\left(\lambda_{1}+\lambda_{2}\right) \check{h}=\lambda_{1} \breve{h} \oplus \lambda_{2} \breve{h}$,

(6) $\check{h}^{\lambda_{1}+\lambda_{2}}=\check{h}^{\lambda_{1}} \otimes \check{h}^{\lambda_{2}}$.

Definition $2.5^{36}$. Let $a_{i}=\left(s_{\theta_{i}}, \ln \left(s_{\theta_{i}}\right)\right)$ be a LIFE, then the score function $s\left(a_{i}\right)$ of $a_{i}$ can be defined as

$$
s\left(a_{i}\right)=\frac{\theta_{i}}{g\left|\operatorname{lh}\left(s_{\theta_{i}}\right)\right|} \sum_{\left(\mu_{i}^{(k)}, v_{i}^{(k)}\right) \in \operatorname{lh}\left(s_{\theta_{i}}\right)}\left(\mu_{i}^{(k)}-v_{i}^{(k)}\right),
$$

and the accuracy function $h\left(a_{i}\right)$ of $a_{i}$ can be defined as

$$
h\left(a_{i}\right)=\frac{\theta_{i}}{g\left|\operatorname{lh}\left(s_{\theta_{i}}\right)\right|} \sum_{\left(\mu_{i}^{(k)}, v_{i}^{(k)}\right) \in \operatorname{lh}\left(s_{\theta_{i}}\right)}\left(\mu_{i}^{(k)}+v_{i}^{(k)}\right),
$$

where $g$ is the number of linguistic arguments in linguistic term set $S$ and $\left|l h\left(s_{\theta_{i}}\right)\right|$ is the number of intuitionistic fuzzy memberships in $\operatorname{lh}\left(s_{\theta_{i}}\right)$.

Based on the score function $s\left(a_{i}\right)$ and the accuracy function $h\left(a_{i}\right)$, we can rank LIFEs as follows. Let $a_{i}=\left(s_{\theta_{i}}, \operatorname{lh}\left(s_{\theta_{i}}\right)\right)$ and $a_{j}=\left(s_{\theta_{j}}, \operatorname{lh}\left(s_{\theta_{j}}\right)\right)$ be two LIFEs, then

(1) If $s\left(a_{i}\right)<s\left(a_{j}\right)$, then $a_{i}<a_{j}$,

(2) If $s\left(a_{i}\right)=s\left(a_{j}\right)$ and $h\left(a_{i}\right)<h\left(a_{j}\right)$, then $a_{i}<$ $a_{j}$, else if $s\left(a_{i}\right)=s\left(a_{j}\right)$ and $h\left(a_{i}\right)=h\left(a_{j}\right)$, then $a_{i} \sim a_{j}$.

Definition 2.6 ${ }^{36}$. Let $\breve{h}=\left\{\left(s_{\theta_{i}}, \operatorname{lh}\left(s_{\theta_{i}}\right)\right)\right\}$ be a LHIFE, the score function $S(\check{h})$ can be defined as

$$
S(\check{h})=\frac{1}{|\check{h}|}\left(\sum \frac{\theta_{i}}{g\left|\operatorname{lh}\left(s_{\theta_{i}}\right)\right|} \sum_{\left(\mu_{i}^{(k)}, v_{i}^{(k)}\right) \in \operatorname{lh}\left(s_{\theta_{i}}\right)}\left(\mu_{i}^{(k)}-v_{i}^{(k)}\right)\right) \text {, }
$$

and the accuracy function $A(\breve{h})$ can be defined as

$$
A(\check{h})=\frac{1}{|\check{h}|}\left(\sum \frac{\theta_{i}}{g\left|\operatorname{lh}\left(s_{\theta_{i}}\right)\right|} \sum_{\left(\mu_{i}^{(k)}, v_{i}^{(k)}\right) \in \operatorname{lh}\left(s_{\theta_{i}}\right)}\left(\mu_{i}^{(k)}+v_{i}^{(k)}\right)\right) \text {, }
$$

where $|\breve{h}|$ is the number of LIFEs in $\breve{h}$ and $g$ is the number of linguistic terms in linguistic term set $S$, $\left|l h\left(s_{\theta_{i}}\right)\right|$ is the number of intuitionistic fuzzy memberships in $\operatorname{lh}\left(s_{\theta_{i}}\right)$.

Based on the score function and accuracy function, we present the following method to compare LHIFEs. Let $\breve{h}_{1}$ and $\check{h}_{2}$ be two LHIFEs,

(1) If $S\left(\breve{h}_{1}\right)<S\left(\breve{h}_{2}\right)$, then $\breve{h}_{1}<\breve{h}_{2}$;

(2) If $S\left(\check{h}_{1}\right)=S\left(\check{h}_{2}\right)$ and

(I) $A\left(\breve{h}_{1}\right)<A\left(\breve{h}_{2}\right)$, then $\check{h}_{1}<\check{h}_{2}$, 
(II) $A\left(\check{h}_{1}\right)=A\left(\breve{h}_{2}\right)$, then $\check{h}_{1} \sim \check{h}_{2}$.

Definition 2.7. Let $\breve{h}_{j}(j=1,2, \ldots, n)$ be a collection of LHIFEs, $w=\left(w_{1}, w_{2}, \ldots, w_{n}\right)$ be the weight vector of $\breve{h}_{j}(j=1,2, \ldots, n)$ with $w_{j} \geqslant 0(j=$ $1,2, \ldots, n)$ and $\sum_{j=1}^{n} w_{j}=1$. The linguistic hesitant intuitionistic fuzzy weighted averaging (LHIFWA) operator is a mapping LHIFWA: $H^{n} \rightarrow H$, which can be defined as follows:

$$
\begin{aligned}
& \operatorname{LHIFWA}_{w}\left(\check{h}_{1}, \check{h}_{2}, \ldots, \check{h}_{n}\right) \\
= & w_{1} \breve{h}_{1} \oplus w_{2} \breve{h}_{2} \oplus \ldots \oplus w_{n} \breve{h}_{n} .
\end{aligned}
$$

Theorem 2. Let $\breve{h}_{j}(j=1,2, \ldots, n)$ be a collection of LHIFEs, $w=\left(w_{1}, w_{2}, \ldots, w_{n}\right)$ be the weight vector of $\breve{h}_{j}(j=1,2, \ldots, n)$ with $w_{j} \geqslant 0(j=$ $1,2, \ldots, n)$ and $\sum_{j=1}^{n} w_{j}=1$. The aggregated result of the LHIFWA operator is also a LHIFE, and

$$
\begin{aligned}
= & \bigcup_{a_{i} \in \breve{h}_{i}}\left\{\left(s_{\left(\sum_{j=1}^{n} w_{j} \theta_{j}\right)}, \bigcup_{\left(\mu_{j}^{(k)}, v_{j}^{(k)}\right) \in \ln \left(s_{\theta_{j}}\right)}\{(1-\right.\right. \\
& \left.\left.\left.\left.\prod_{j=1}^{n}\left(1-\mu_{j}^{(k)}\right)^{w_{j}}, \prod_{j=1}^{n}\left(v_{j}^{(k)}\right)^{w_{j}}\right)\right\}\right)\right\},
\end{aligned}
$$

where $a_{i}=\left\{\left(s_{\theta_{i}}, \operatorname{lh}\left(s_{\theta_{i}}\right)\right)\right\},\left(\mu_{j}^{(k)}, v_{j}^{(k)}\right) \in \operatorname{lh}\left(s_{\theta_{j}}\right), k=$ $1,2, \ldots, l_{j}, l_{j}$ is the number of intuitionistic fuzzy memberships in $\operatorname{lh}\left(s_{\theta_{j}}\right)$.

Definition 2.8. Let $\breve{h}_{j}(j=1,2, \ldots, n)$ be a collection of LHIFEs, $w=\left(w_{1}, w_{2}, \ldots, w_{n}\right)$ be the weight vector of $\breve{h}_{j}(j=1,2, \ldots, n)$ with $w_{j} \geqslant 0(j=$ $1,2, \ldots, n)$ and $\sum_{j=1}^{n} w_{j}=1$. The linguistic hesitant intuitionistic fuzzy weighted geometric (LHIFWG) operator is a mapping LHIFWG: $H^{n} \rightarrow H$, which can be defined as follows:

$$
\begin{aligned}
& \operatorname{LHIFWG}_{w}\left(\breve{h}_{1}, \breve{h}_{2}, \ldots, \breve{h}_{n}\right) \\
= & \breve{h}_{1}^{w_{1}} \otimes \breve{h}_{2}^{w_{2}} \otimes \ldots \otimes \breve{h}_{n}^{w_{n}} .
\end{aligned}
$$

Theorem 3. Let $\breve{h}_{j}(j=1,2, \ldots, n)$ be a collection of LHIFEs, $w=\left(w_{1}, w_{2}, \ldots, w_{n}\right)$ be the weight vector of $\breve{h}_{j}(j=1,2, \ldots, n)$ with $w_{j} \geqslant 0(j=$ $1,2, \ldots, n)$ and $\sum_{j=1}^{n} w_{j}=1$. The aggregated result of the LHIFWG operator is also a LHIFE, and

$$
\begin{aligned}
= & \bigcup_{a_{i} \in \breve{h}_{i}}\left\{\left(s_{\left(\prod_{j=1}^{n}\left(\theta_{j}\right)^{w_{j}}\right)}, \bigcup_{\left(\mu_{j}^{(k)}, v_{j}^{(k)}\right) \in \operatorname{lh}\left(s_{\theta_{j}}\right)}\right.\right. \\
& \left.\left.\left\{\left(\prod_{j=1}^{n}\left(\mu_{j}^{(k)}\right)^{w_{j}}, 1-\prod_{j=1}^{n}\left(1-v_{j}^{(k)}\right)^{w_{j}}\right)\right\}\right)\right\},
\end{aligned}
$$

where $a_{i}=\left\{\left(s_{\theta_{i}}, \operatorname{lh}\left(s_{\theta_{i}}\right)\right)\right\},\left(\mu_{j}^{(k)}, v_{j}^{(k)}\right) \in \operatorname{lh}\left(s_{\theta_{j}}\right), k=$ $1,2, \ldots, l_{j}, l_{j}$ is the number of intuitionistic fuzzy memberships in $\operatorname{lh}\left(s_{\theta_{j}}\right)$.

Definition 2.9. Let $\check{h}_{j}(j=1,2, \ldots, n)$ be a collection of LHIFEs, $w=\left(w_{1}, w_{2}, \ldots, w_{n}\right)$ be the weight vector of $\breve{h}_{j}(j=1,2, \ldots, n)$ with $w_{j} \geqslant 0(j=$ $1,2, \ldots, n)$ and $\sum_{j=1}^{n} w_{j}=1, \lambda>0$. The generalized linguistic hesitant intuitionistic fuzzy weighted averaging (GLHIFWA) operator is a mapping GLHIFWA: $H^{n} \rightarrow H$, which can be defined as follows:

$$
\begin{aligned}
& \operatorname{GLHIFWA}_{w, \lambda}\left(\check{h}_{1}, \breve{h}_{2}, \ldots, \breve{h}_{n}\right) \\
& =\left(w_{1} \breve{h}_{1}^{\lambda} \oplus w_{2} \breve{h}_{2}^{\lambda} \oplus \ldots \oplus w_{n} \breve{h}_{n}^{\lambda}\right)^{1 / \lambda} .
\end{aligned}
$$

Theorem 4. Let $\check{h}_{j}(j=1,2, \ldots, n)$ be a collection of LHIFEs, $w=\left(w_{1}, w_{2}, \ldots, w_{n}\right)$ be the weight vector of $\breve{h}_{j}(j=1,2, \ldots, n)$ with $w_{j} \geqslant 0(j=$ $1,2, \ldots, n)$ and $\sum_{j=1}^{n} w_{j}=1$. The aggregated result of the GLHIFWA operator is also a LHIFE, and

$$
\begin{aligned}
& \operatorname{GLHIFWA}_{w, \lambda}\left(\check{h}_{1}, \check{h}_{2}, \ldots, \check{h}_{n}\right)
\end{aligned}
$$

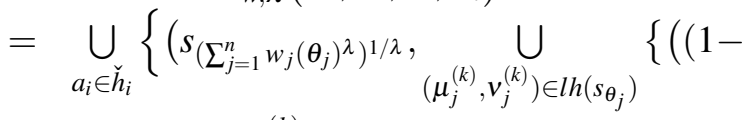

$$
\begin{aligned}
& \left.\prod_{j=1}^{n}\left(1-\left(\mu_{j}^{(k)}\right)^{\lambda}\right)^{w_{j}}\right)^{1 / \lambda}, 1-\left(1-\prod_{j=1}^{n}(1-\right. \\
& \left.\left.\left.\left.\left.\left.\left(1-v_{j}^{(k)}\right)^{\lambda}\right)^{w_{j}}\right)^{1 / \lambda}\right)\right\}\right)\right\} \text {. }
\end{aligned}
$$

where $a_{i}=\left\{\left(s_{\theta_{i}}, \operatorname{lh}\left(s_{\theta_{i}}\right)\right) \in \breve{h}_{i}\right\}, \quad\left(\mu_{j}^{(k)}, v_{j}^{(k)}\right) \in$ $\operatorname{lh}\left(s_{\theta_{j}}\right), k=1,2, \ldots, l_{j}$ and $l_{j}$ is the number of intuitionistic fuzzy memberships in $\operatorname{lh}\left(s_{\theta_{j}}\right), \lambda>0$.

\section{Cross-entropy measures for LHIFSs}

Let $P=\left\{p_{1}, p_{2}, \ldots, p_{n}\right\}$ and $Q=\left\{q_{1}, q_{2}, \ldots, q_{n}\right\}$ be two probability distribution. In order to measure the divergence between $P$ and $Q$, Kullback and Leibler ${ }^{39}$ defined the cross-entropy measure as

$$
C E_{1}(P, Q)=\sum_{i=1}^{n} p_{i} \ln \frac{p_{i}}{q_{i}}
$$

If $n=2, P=\{p, 1-p\}, Q=\{q, 1-q\}$, then $C E_{1}(P, Q)=p \ln \frac{p}{q}+(1-p) \ln \frac{1-p}{1-q}$.

Bhandari and $\mathrm{Pal}^{40}$ generalized the cross-entropy measure based on probability distribution to accommodate fuzzy information. Let $A$ and $B$ be two fuzzy 
sets in the finite universe $X=\left\{x_{1}, x_{2}, \ldots, x_{n}\right\}$, then the cross-entropy measure for fuzzy values can be defined as

$$
\begin{aligned}
C E_{2}(A, B)= & \sum_{i=1}^{n}\left(\mu_{A}\left(x_{i}\right) \ln \frac{\mu_{A}\left(x_{i}\right)}{\mu_{B}\left(x_{i}\right)}+\right. \\
& \left.\left(1-\mu_{A}\left(x_{i}\right)\right) \ln \frac{1-\mu_{A}\left(x_{i}\right)}{1-\mu_{B}\left(x_{i}\right)}\right) .
\end{aligned}
$$

Vlachos and Sergiadis ${ }^{49}$ developed a crossentropy measure for intuitionistic fuzzy information by extending fuzzy cross-entropy measure to intuitionistic fuzzy environment. Let $A^{\prime}$ and $B^{\prime}$ be two intuitionistic fuzzy sets in the finite universe $X=\left\{x_{1}, x_{2}, \ldots, x_{n}\right\}$, then the cross-entropy measure for intuitionsitic fuzzy values can be defined as

$C E_{3}\left(A^{\prime}, B^{\prime}\right)=\sum_{i=1}^{n}\left(\mu_{A}\left(x_{i}\right) \ln \frac{\mu_{A}\left(x_{i}\right)}{\mu_{B}\left(x_{i}\right)}+v_{A}\left(x_{i}\right) \ln \frac{v_{A}\left(x_{i}\right)}{v_{B}\left(x_{i}\right)}\right)$.

If $\mu_{B}\left(x_{i}\right)=0, \mu_{A}\left(x_{i}\right) \neq 0$ or $v_{B}\left(x_{i}\right)=0, v_{A}\left(x_{i}\right) \neq 0$, $C E_{3}\left(A^{\prime}, B^{\prime}\right)$ is undefined. Vlachos and Sergiadis further gave the following cross-entropy

$$
\begin{aligned}
C E_{4}\left(A^{\prime}, B^{\prime}\right)= & \sum_{i=1}^{n}\left(\mu_{A}\left(x_{i}\right) \ln \frac{\mu_{A}\left(x_{i}\right)}{\frac{1}{2}\left(\mu_{A}\left(x_{i}\right)+\mu_{B}\left(x_{i}\right)\right)}+\right. \\
& \left.v_{A}\left(x_{i}\right) \ln \frac{v_{A}\left(x_{i}\right)}{\frac{1}{2}\left(v_{A}\left(x_{i}\right)+v_{B}\left(x_{i}\right)\right)}\right) .
\end{aligned}
$$

Uncertainty of intuitionistic fuzzy values is decomposed into intuitionism and fuzziness. Intuitionism is determined by hesitancy degree $\pi_{A}\left(x_{i}\right)=$ $1-\mu_{A}\left(x_{i}\right)-v_{A}\left(x_{i}\right)$ and fuzziness is determined by the closeness of membership $\mu_{A}\left(x_{i}\right)$ and nonmembership $v_{A}\left(x_{i}\right)$ as $\Delta_{A}\left(x_{i}\right)=\left|\mu_{A}\left(x_{i}\right)-v_{A}\left(x_{i}\right)\right|$. Mao et al. ${ }^{43}$ presented the cross-entropy measure for intuitionistic fuzzy information by considering intuitionism and fuzziness simultaneously as follows

$$
\begin{aligned}
C E_{5}\left(A^{\prime}, B^{\prime}\right)= & \sum_{i=1}^{n}\left(\pi_{A}\left(x_{i}\right) \ln \frac{\pi_{A}\left(x_{i}\right)}{\frac{1}{2}\left(\pi_{A}\left(x_{i}\right)+\pi_{B}\left(x_{i}\right)\right)}+\right. \\
& \left.\triangle_{A}\left(x_{i}\right) \ln \frac{\triangle_{A}\left(x_{i}\right)}{\frac{1}{2}\left(\triangle_{A}\left(x_{i}\right)+\triangle_{B}\left(x_{i}\right)\right)}\right)
\end{aligned}
$$

$\mathrm{Xu}$ and $\mathrm{Xia}^{48}$ proposed some cross-entropy formulas for hesitant fuzzy information by using two concave-up functions $f(x)=(1+q x) \ln (1+q x)$ and $g(x)=x^{p}$. Let $\widetilde{A}=\left\{\widetilde{\alpha}_{1}, \widetilde{\alpha}_{2}, \ldots, \widetilde{\alpha}_{n}\right\}$ and $\widetilde{B}=$ $\left\{\widetilde{\beta}_{1}, \widetilde{\beta}_{2}, \ldots, \widetilde{\beta}_{n}\right\}$ be two hesitant fuzzy sets in the finite universe $X=\left\{x_{1}, x_{2}, \ldots, x_{n}\right\}$. The number of elements in all $\widetilde{\alpha}_{i}(i=1,2, \ldots, n)$ and $\widetilde{\beta}_{i}(i=1,2, \ldots, n)$ is the same. $\left|\widetilde{\alpha}_{i}\right|$ is the number of elements in $\widetilde{\alpha}_{i}$. $\widetilde{\alpha}_{\sigma(i)}$ is the $i$ th largest values in $\widetilde{\alpha}_{i}$. Then the crossentropy measures can be defined as follows in Eq. (12) and Eq.(13). Here $l=\left|\widetilde{\alpha}_{i}\right|, T=(1+q) \ln (1+$ $q)-(2+q)(\ln (2+q)-\ln 2), q>0$.

$$
\begin{aligned}
& C E_{5}(\widetilde{A}, \widetilde{B})=\frac{1}{l T} \sum_{i=1}^{n} \sum_{j=1}^{l}\left(\frac{\left(1+q \widetilde{\alpha}_{\sigma(j)}\left(x_{i}\right)\right) \ln \left(1+q \widetilde{\alpha}_{\sigma(j)}\left(x_{i}\right)\right)+\left(1+q \widetilde{\beta}_{\sigma(j)}\left(x_{i}\right)\right) \ln \left(1+q \widetilde{\beta}_{\sigma(j)}\left(x_{i}\right)\right)}{2}-\right. \\
& \frac{\left(2+q \widetilde{\alpha}_{\sigma(j)}\left(x_{i}\right)+q \widetilde{\beta}_{\sigma(j)}\left(x_{i}\right)\right)}{2} \ln \frac{\left(2+q \widetilde{\alpha}_{\sigma(j)}\left(x_{i}\right)+q \widetilde{\beta}_{\sigma(j)}\left(x_{i}\right)\right)}{2}+ \\
& \frac{\left(1+q\left(1-\widetilde{\alpha}_{\sigma(l-i+1)}\left(x_{i}\right)\right) \ln \left(1+q\left(1-\widetilde{\alpha}_{\sigma(l-j+1)}\left(x_{i}\right)\right)+\left(1+q\left(1-\widetilde{\beta}_{2 \sigma(l-j+1)}\left(x_{i}\right)\right) \ln \left(1+q\left(1-\widetilde{\beta}_{\sigma(l-j+1)}\left(x_{i}\right)\right)\right.\right.\right.\right.}{2} \\
& \left.-\frac{\left.2+q\left(1-\widetilde{\alpha}_{\sigma(l-i+1)}\left(x_{i}\right)\right)+1-\widetilde{\beta}_{\sigma(l-i+1)}\left(x_{i}\right)\right)}{2} \ln \frac{\left.2+q\left(1-\widetilde{\alpha}_{\sigma(l-i+1)}\left(x_{i}\right)\right)+1-\widetilde{\beta}_{\sigma(l-i+1)}\left(x_{i}\right)\right)}{2}\right) . \\
& C E_{6}(\widetilde{A}, \widetilde{B})=\frac{1}{l\left(1-2^{1-p}\right)} \sum_{i=1}^{n} \sum_{j=1}^{l}\left(\frac{\widetilde{\alpha}_{\sigma(j)}^{p}\left(x_{i}\right)+\widetilde{\beta}_{\sigma(j)}^{p}\left(x_{i}\right)}{2}+\frac{\left(1-\widetilde{\alpha}_{\sigma(l-j+1)}\left(x_{i}\right)\right)^{p}+\left(1-\widetilde{\beta}_{\sigma(l-j+1)}\left(x_{i}\right)\right)^{p}}{2}-\right. \\
& \left.\left(\frac{\widetilde{\alpha}_{\sigma(j)}\left(x_{i}\right)+\widetilde{\beta}_{\sigma(j)}\left(x_{i}\right)}{2}\right)^{p}+\frac{\left(1-\widetilde{\alpha}_{\sigma(l-j+1)}\left(x_{i}\right)+1-\widetilde{\beta}_{\sigma(l-j+1)}\left(x_{i}\right)\right)^{p}}{2}\right), l=\left|\widetilde{\alpha}_{i}\right| .
\end{aligned}
$$

Peng et al. ${ }^{26}$ developed several cross-entropy measures for intuitionistic hesitant fuzzy numbers (IHFNs) by extending cross-entropy measures for hesitant fuzzy elements introduced by $\mathrm{Xu}$ and $\mathrm{Xia}^{48}$. Let $\widetilde{\alpha}_{j}=<\Gamma_{\widetilde{\alpha}_{j}}, \Psi_{\widetilde{\alpha}_{j}}>(j=1,2)$ be IHFNs, $\Gamma_{\widetilde{\alpha}_{j}}$ and $\Psi_{\widetilde{\alpha}_{j}}$ denote the possible degrees of membership and non-membership, respectively. $\Pi_{\widetilde{\alpha}_{j}}$ denotes the possible degrees of hesitation. Here $T=$ $(1+q) \ln (1+q)-(2+q)(\ln (2+q)-\ln 2), q>0$. 


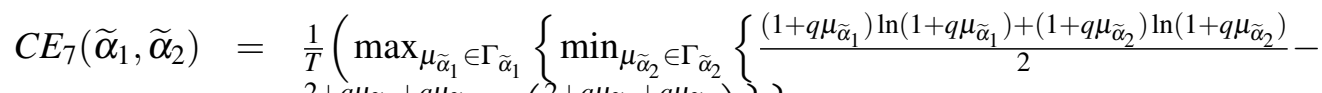

$$
\begin{aligned}
& \left.\left.\frac{2+q \mu_{\widetilde{\alpha}_{1}}+q \mu_{\widetilde{\alpha}_{2}}}{2} \ln \left(\frac{2+q \mu_{\widetilde{\alpha}_{1}}+q \mu_{\widetilde{\alpha}_{2}}}{2}\right)\right\}\right\}+
\end{aligned}
$$

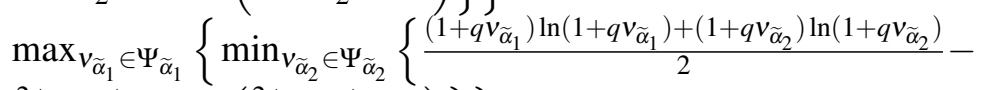

$$
\begin{aligned}
& \left.\left.\frac{2+q v_{\widetilde{\alpha}_{1}}+q v_{\widetilde{\alpha}_{2}}}{2} \ln \left(\frac{2+q v_{\widetilde{\alpha}_{1}}+q v_{\widetilde{\alpha}_{2}}}{2}\right)\right\}\right\}+
\end{aligned}
$$

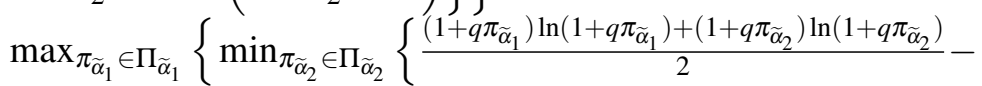

$$
\begin{aligned}
& \left.\left.\left.\frac{2+q \pi_{\widetilde{\alpha}_{1}}+q \pi_{\widetilde{\alpha}_{2}}}{2} \ln \left(\frac{2+q \pi_{\widetilde{\alpha}_{1}}+q \pi_{\tilde{\alpha}_{2}}}{2}\right)\right\}\right\}\right), p \geqslant 1 \text {. }
\end{aligned}
$$

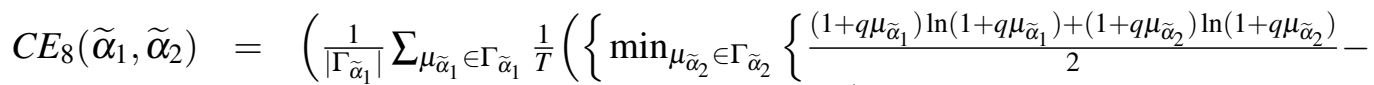

$$
\begin{aligned}
& \left.\left.\left.\frac{2+q \mu_{\widetilde{\alpha}_{1}}+q \mu_{\widetilde{\alpha}_{2}}}{2} \ln \left(\frac{2+q \mu_{\widetilde{\alpha}_{1}}+q \mu_{\widetilde{\alpha}_{2}}}{2}\right)\right\}\right)^{p}\right)^{1 / p}+
\end{aligned}
$$

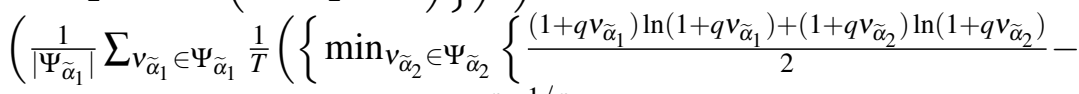

$$
\begin{aligned}
& \left.\left.\left.\frac{2+q v_{\widetilde{\alpha}_{1}}+q v_{\widetilde{\alpha}_{2}}}{2} \ln \left(\frac{2+q v_{\widetilde{\alpha}_{1}}+q v_{\widetilde{\alpha}_{2}}}{2}\right)\right\}\right)^{p}\right)^{1 / p}
\end{aligned}
$$

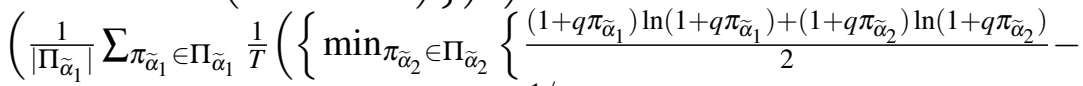

$$
\begin{aligned}
& \left.\left.\left.\frac{2+q \pi_{\tilde{\alpha}_{1}}+q \pi_{\tilde{\alpha}_{2}}}{2} \ln \left(\frac{2+q \pi_{\widetilde{\alpha}_{1}}+q \pi_{\tilde{\alpha}_{2}}}{2}\right)\right\}\right)^{p}\right)^{1 / p}, p \geqslant 1 \text {. }
\end{aligned}
$$

In the following, we propose the axiomatic definition of cross-entropy measure for linguistic hesitant intuitionistic fuzzy information as follows, motivated by $\mathrm{Xu}$ and $\mathrm{Xia}{ }^{48}$, Peng et al. ${ }^{26}$, etc.

Definition 3.1. Let $\breve{h}_{1}, \breve{h}_{2} \in H, C E^{\prime}: H \times H \rightarrow$ $R^{+}$, then cross-entropy $C E^{\prime}\left(\breve{h}_{1}, \breve{h}_{2}\right)$ of $\breve{h}_{1}$ and $\check{h}_{2}$ should satisfy the following conditions:

(1) $C E^{\prime}\left(\check{h}_{1}, \breve{h}_{2}\right) \geqslant 0, \forall \check{h}_{1}, \check{h}_{2} \in H$,

(2) $C E^{\prime}\left(\breve{h}_{1}, \breve{h}_{2}\right)=0$, if $\breve{h}_{1}=\breve{h}_{2}$,

(3) $C E^{\prime}\left(\check{h}_{1}^{c}, \breve{h}_{2}^{c}\right)=C E^{\prime}\left(\check{h}_{1}, \breve{h}_{2}\right), \forall \breve{h}_{1}, \check{h}_{2} \in H$.

Here $\check{h}_{i}^{c}=\left\{\left(s_{\left(g-\theta_{i}\right)}, \quad \operatorname{lh}\left(s_{\theta_{i}}\right)^{c}\right)\right\}, \operatorname{lh}\left(s_{\theta_{i}}\right)^{c}=$ $\left\{\left(v_{i}^{(k)}, \mu_{i}^{(k)}\right)\right\},\left(\mu_{i}^{(k)}, v_{i}^{(k)}\right) \in \operatorname{lh}\left(s_{\theta_{i}}\right), i=1,2$.

We develop several cross-entropy measures for linguistic hesitant intuitionistic fuzzy information, motivated by Vlachos and Sergiadis ${ }^{49}$, Mao et al. ${ }^{43}$, $\mathrm{Xu}$ and $\mathrm{Xia}^{48}$, Peng et al. ${ }^{26}$, etc.

Let $\check{h}_{1}=\left\{a_{1 i}\right\}=\left\{\left(s_{\theta_{1 i}}, \operatorname{lh}\left(s_{\theta_{1 i}}\right)\right)\right\},\left(\mu_{1 i}^{(k)}, v_{1 i}^{(k)}\right) \in$ $\operatorname{lh}\left(s_{\theta_{1 i}}\right), \quad \check{h}_{2}=\left\{a_{2 j}\right\}=\left\{\left(s_{\theta_{2 j}}, \operatorname{lh}\left(s_{\theta_{2 j}}\right)\right)\right\}$, $\left(\mu_{2 j}^{(k)}, v_{2 j}^{(k)}\right) \in \operatorname{lh}\left(s_{\theta_{2 j}}\right)$. Then two cross entropy measures can be defined as follows by considering linguistic variables, intuitionistic fuzzy memberships. Since each LHIFE has several linguistic terms and intuitionistic fuzzy memberships, we can define cross-entropy measures by considering the maxi- mum value and the average value.

$$
\begin{aligned}
& C E_{1}^{\prime}\left(\check{h}_{1}, \check{h}_{2}\right) \\
= & \max _{a_{k i} \in \breve{h}_{k}}\left\{\frac{\theta_{1 \sigma(i)}}{g} \log _{2} \frac{2 \theta_{1 \sigma(i)}}{\theta_{1 \sigma(i)}+\theta_{2 \sigma(i)}}+\right. \\
& \left.\left(1-\frac{\theta_{1 \sigma(i)}}{g}\right) \log _{2} \frac{2\left(g-\theta_{1 \sigma(i)}\right)}{2 g-\theta_{1 \sigma(i)}-\theta_{2 \sigma(i)}}\right\}+ \\
& \max _{\left(\mu_{i j}^{(k)}, v_{i j}^{(k)}\right) \in \operatorname{lh}\left(s_{\theta_{i j}}\right)}\left\{\mu_{1 \sigma(j)}^{(k)} \log _{2} \frac{2 \mu_{1 \sigma(j)}^{(k)}}{\mu_{1 \sigma(j)}^{(k)}+\mu_{2 \sigma(j)}^{(k)}}+\right. \\
& \left.v_{1 \sigma(j)}^{(k)} \log _{2} \frac{2 v_{1 \sigma(j)}^{(k)}}{v_{1 \sigma(j)}^{(k)}+v_{2 \sigma(j)}^{(k)}}\right\} .
\end{aligned}
$$

$$
\begin{aligned}
& C E_{2}^{\prime}\left(\check{h}_{1}, \check{h}_{2}\right) \\
& =\frac{1}{\left|a_{1 i}\right|} \sum_{a_{k i} \in \check{h}_{k}}\left(\left(\frac{\theta_{1 \sigma(i)}}{g} \log _{2} \frac{2 \theta_{1 \sigma(i)}}{\theta_{1 \sigma(i)}+\theta_{2 \sigma(i)}}+\right.\right. \\
& \left.\left(1-\frac{\theta_{1 \sigma(i)}}{g}\right) \log _{2} \frac{2\left(g-\theta_{1 \sigma(i)}\right)}{2 g-\theta_{1 \sigma(i)}-\theta_{2 \sigma(i)}}\right) \\
& \frac{1}{\left|l h\left(s_{\left(\theta_{k i}\right)}\right)\right|} \sum_{\left(\mu_{i j}^{(k)}, v_{i j}^{(k)}\right) \in \operatorname{lh}\left(s_{\theta_{i j}}\right)}\left(\mu_{1 \sigma(j)}^{(k)} \log _{2} \frac{2 \mu_{1 \sigma(j)}^{(k)}}{\mu_{1 \sigma(j)}^{(k)}+\mu_{2 \sigma(j)}^{(k)}}\right. \\
& \left.\left.+v_{1 \sigma(j)}^{(k)} \log _{2} \frac{2 v_{1 \sigma(j)}^{(k)}}{v_{1 \sigma(j)}^{(k)}+v_{2 \sigma(j)}^{(k)}}\right)\right) \text {. }
\end{aligned}
$$

Similarly, other cross-entropy measures for linguistic hesitant intuitionistic fuzzy elements can be 
defined as follows. By using the generalized mean operator, we can get the cross-entropy measures $C E_{3}^{\prime}\left(\breve{h}_{1}, \breve{h}_{2}\right)$ and $C E_{4}^{\prime}\left(\breve{h}_{1}, \breve{h}_{2}\right)$.

$$
\begin{aligned}
& C E_{3}^{\prime}\left(\breve{h}_{1}, \breve{h}_{2}\right) \\
& =\left(\frac { 1 } { | a _ { 1 i } | } \sum _ { a _ { k i } \check { h } _ { k } } \left(\left(\frac{\theta_{1 \sigma(i)}}{g} \log _{2} \frac{2 \theta_{1 \sigma(i)}}{\theta_{1 \sigma(i)}+\theta_{2 \sigma(i)}}\right)^{p}+\right.\right. \\
& \left(\left(1-\frac{\theta_{1 \sigma(i)}}{g}\right) \log _{2} \frac{2\left(g-\theta_{1 \sigma(i)}\right)}{2 g-\theta_{1 \sigma(i)}-\theta_{2 \sigma(i)}}\right)^{p} \\
& +\frac{1}{\left|l h\left(s_{\left.\theta_{k i}\right)}\right)\right|} \sum_{\left(\mu_{i j}^{(k)}, v_{i j}^{(k)}\right) \in \operatorname{lh}\left(s_{\left.\theta_{i j}\right)}\right.}\left(\left(\mu_{1 \sigma(j)}^{(k)} \log _{2} \frac{2 \mu_{1 \sigma(j)}^{(k)}}{\mu_{1 \sigma(j)}^{(k)}+\mu_{2 \sigma(j)}^{(k)}}\right)^{p}\right. \\
& \left.\left.+\left(v_{1 \sigma(j)}^{(k)} \log _{2} \frac{2 v_{1 \sigma(j)}^{(k)}}{v_{1 \sigma(j)}^{(k)}+v_{2 \sigma(j)}^{(k)}}\right)^{p}\right)\right)^{1 / p}, p \geqslant 1 .
\end{aligned}
$$

$\mathrm{Ye}^{38}$ developed the cross-entropy measure for intuitionistic fuzzy value by considering the complementary set of the intuitionistic fuzzy set to get

$$
\begin{aligned}
& C E\left(A^{\prime}, B^{\prime}\right) \\
= & \sum_{i=1}^{n}\left(\frac{\mu_{A}\left(x_{i}\right)+1-v_{A}\left(x_{i}\right)}{2} *\right. \\
& \log _{2} \frac{1}{\left.\frac{1}{2}\left[\left(\mu_{A}\left(x_{i}\right)+1-v_{A}\right)+1-v_{A}\left(x_{i}\right)\right)+\mu_{B}\left(x_{i}\right)+1-v_{B}\left(x_{i}\right)\right]}+ \\
& \frac{1-\mu_{A}\left(x_{i}\right)+v_{A}\left(x_{i}\right)}{2} * \\
& \left.\log _{2} \frac{1-\mu_{A}\left(x_{i}\right)+v_{A}\left(x_{i}\right)}{\frac{1}{2}\left[\left(1-\mu_{A}\left(x_{i}\right)+v_{A}\left(x_{i}\right)\right)+1-\mu_{B}\left(x_{i}\right)+v_{B}\left(x_{i}\right)\right]}\right) .
\end{aligned}
$$

We extend the cross-entropy measure $C E\left(A^{\prime}, B^{\prime}\right)$ in $\mathrm{Ye}^{38}$ to accommodate linguistic hesitant intuitionitic fuzzy values to get cross-entropy measures $C E_{5}^{\prime}\left(\breve{h}_{1}, \breve{h}_{2}\right)$ and $C E_{6}^{\prime}\left(\breve{h}_{1}, \breve{h}_{2}\right)$

$$
\begin{aligned}
& C E_{4}^{\prime}\left(\check{h}_{1}, \breve{h}_{2}\right) \\
= & \left(\frac{1}{\left|a_{1 i}\right|} \sum_{a_{k i} \in \check{h}_{k}}\left(\frac{\theta_{1 \sigma(i)}}{g} \log _{2} \frac{2 \theta_{1 \sigma(i)}}{\theta_{1 \sigma(i)}+\theta_{2 \sigma(i)}}\right)^{p}\right)^{1 / p}+ \\
& \left(\frac{1}{\left|a_{1 i}\right|} \sum_{a_{k i} \in \check{h}_{k}}\left(\left(1-\frac{\theta_{1 \sigma(i)}}{g}\right) \log _{2} \frac{2\left(g-\theta_{1 \sigma(i)}\right)}{2 g-\theta_{1 \sigma(i)}-\theta_{2 \sigma(i)}}\right)^{p}\right)^{1 / p} \\
& +\left(\frac{1}{\left|a_{1 i}\right|\left|l h\left(s_{\left(\theta_{k i}\right)}\right)\right|} \sum_{\left(\mu_{i j}^{(k)}, v_{i j}^{(k)}\right) \in \operatorname{lh}\left(s _ { \theta _ { i j } ) } \left(\mu_{i \sigma(j)}^{(k)} *\right.\right.}\right. \\
& \left.\left.\log _{2} \frac{2 \mu_{1 \sigma(j)}^{(k)}}{\mu_{1 \sigma(j)}^{(k)}+\mu_{2 \sigma(j)}^{(k)}}\right)^{p}\right)^{1 / p}+\left(\frac{1}{\left|a_{1 i}\right| \mid l h\left(s_{\left.\theta_{k i}\right)} \mid\right.} \sum_{\left(\mu_{i j}^{(k)}, v_{i j}^{(k)}\right) \in \operatorname{lh}\left(s_{\theta_{i j}}\right)}\right. \\
& \left.\left(v_{i \sigma(j)}^{(k)} \log _{2} \frac{2 v_{1 \sigma(j)}^{(k)}}{v_{1 \sigma(j)}^{(k)}+v_{2 \sigma(j)}^{(k)}}\right)^{p}\right)^{1 / p}, p \geqslant 1 .
\end{aligned}
$$

$$
\begin{aligned}
& C E_{5}^{\prime}\left(\check{h}_{1}, \check{h}_{2}\right) \\
& =\max _{a_{k i} \in \breve{h}_{k}}\left\{\frac{\theta_{1 \sigma(i)}}{g} \log _{2} \frac{2 \theta_{1 \sigma(i)}}{\theta_{1 \sigma(i)}+\theta_{2 \sigma(i)}}+\right. \\
& \left.\left(1-\frac{\theta_{1 \sigma(i)}}{g}\right) \log _{2} \frac{2\left(g-\theta_{1 \sigma(i)}\right)}{2 g-\theta_{1 \sigma(i)}-\theta_{2 \sigma(i)}}\right\}+ \\
& \max _{\left(\mu_{i j}^{(k)}, v_{i j}^{(k)}\right) \in \operatorname{lh}\left(s_{\theta_{i j}}\right)}\left\{\frac{1+\mu_{1 \sigma(j)}^{(k)}-v_{1 \sigma(j)}^{(k)}}{2} *\right. \\
& \log _{2} \frac{2\left(1+\mu_{1 \sigma(j)}^{(k)}-v_{1 \sigma(j)}^{(k)}\right)}{2+\mu_{1 \sigma(j)}^{(k)}-v_{1 \sigma(j)}^{(k)}+\mu_{2 \sigma(j)}^{(k)}-v_{2 \sigma(j)}^{(k)}}+ \\
& \left.\frac{1-\mu_{1 \sigma(j)}^{(k)}+v_{1 \sigma(j)}^{(k)}}{2} \log _{2} \frac{2\left(1-\mu_{1 \sigma(j)}^{(k)}+v_{1 \sigma(j)}^{(k)}\right)}{2-\mu_{1 \sigma(j)}^{(k)}+v_{1 \sigma(j)}^{(k)}-\mu_{2 \sigma(j)}^{(k)}+v_{2 \sigma(j)}^{(k)}}\right\} \text {. }
\end{aligned}
$$

$$
\begin{aligned}
& C E_{6}^{\prime}\left(\breve{h}_{1}, \breve{h}_{2}\right)=\left(\frac{1}{\left|a_{1 i}\right|} \sum_{a_{k i} \in \breve{h}_{k}}\left(\frac{\theta_{1 \sigma(i)}}{g} \log _{2} \frac{2 \theta_{1 \sigma(i)}}{\theta_{1 \sigma(i)}+\theta_{2 \sigma(i)}}\right)^{p}\right)^{1 / p}+\left(\frac{1}{\left|a_{1 i}\right|} \sum_{a_{1 i} \in \check{h}_{1}}\left(\left(1-\frac{\theta_{1 \sigma(i)}}{g}\right) \log _{2} \frac{2\left(g-\theta_{1 \sigma(i)}\right)}{2 g-\theta_{1 \sigma(i)}-\theta_{2 \sigma(i)}}\right)^{p}\right)^{1 / p} \\
& \left(\frac{1}{\left|a_{1 i}\right|\left|l h\left(s_{\left(\theta_{k i}\right)}\right)\right|} \sum_{\left(\mu_{i j}^{(k)}, v_{i j}^{(k)}\right) \in \ln \left(s_{\left.\theta_{i j}\right)}\right.}\left(\frac{\mu_{1 \sigma(j)}^{(k)}+1-v_{1 \sigma(j)}^{(k)}}{2} \log _{2} \frac{2\left(\mu_{1 \sigma(j)}^{(k)}+1-v_{1 \sigma(j)}^{(k)}\right)}{2+\mu_{1 \sigma(j)}^{(k)}-v_{1 \sigma(j)}^{(k)}+\mu_{2 \sigma(j)}^{(k)}-v_{2 \sigma(j)}^{(k)}}\right)^{p}\right)^{1 / p}+
\end{aligned}
$$

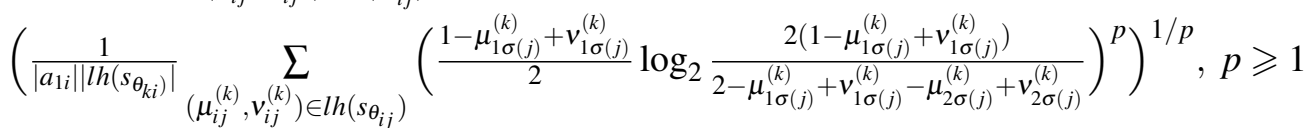

By using the concave-up function $f(x)=x^{p}$, we extend the cross-entropy $C E_{6}(\widetilde{A}, \widetilde{B})$ developed by $\mathrm{Xu}$ and $\mathrm{Xia}^{48}$ to linguistic hesitant intuitionis- tic fuzzy environment to get $C E_{7}^{\prime}\left(\check{h}_{1}, \check{h}_{2}\right)$. By using the concave-up function $f(x)=x^{p}$ and the generalized mean operator, we can further get cross-entropy measure $C E_{8}^{\prime}\left(\breve{h}_{1}, \breve{h}_{2}\right)$. 


$$
\begin{aligned}
& C E_{7}^{\prime}\left(\breve{h}_{1}, \breve{h}_{2}\right)=\frac{1}{1-2^{1-p}}\left(\max _{a_{k i} \in \breve{h}_{k}}\left\{\frac{\left(\theta_{1 \sigma(i)} / g\right)^{p}+\left(\theta_{2 \sigma(i)} / g\right)^{p}}{2}-\left(\frac{\theta_{1 \sigma(i)} / g+\theta_{2 \sigma(i)} / g}{2}\right)^{p}\right\}+\right. \\
& \max _{a_{k i} \in \breve{h}_{k}}\left\{\frac{\left(1-\theta_{1 \sigma(i)} / g\right)^{p}+\left(1-\theta_{2 \sigma(i)} / g\right)^{p}}{2}-\left(1-\frac{\theta_{1 \sigma(i)} / g+\theta_{2 \sigma(i)} / g}{2}\right)^{p}\right\} \\
& +\max _{\left(\mu_{i j}^{(k)}, v_{i j}^{(k)}\right) \in \operatorname{lh}\left(s_{\theta_{i j}}\right)}\left\{\frac{\left(\mu_{1 \sigma(j)}^{(k)}\right)^{p}+\left(\mu_{2 \sigma(j)}^{(k)}\right)^{p}}{2}-\left(\frac{\mu_{1 \sigma(j)}^{(k)}+\mu_{2 \sigma(j)}^{(k)}}{2}\right)^{p}\right\}+ \\
& \left.\max _{\left(\mu_{i j}^{(k)}, v_{i j}^{(k)}\right) \in \operatorname{lh}\left(s_{\theta_{i j}}\right)}\left\{\frac{\left(v_{1 \sigma(j)}^{(k)}\right)^{p}+\left(v_{2 \sigma(j)}^{(k)}\right)^{p}}{2}-\left(\frac{v_{1 \sigma(j)}^{(k)}+v_{2 \sigma(j)}^{(k)}}{2}\right)^{p}\right\}\right), 2 \geqslant p>1 ;
\end{aligned}
$$

$$
\begin{aligned}
& C E_{8}^{\prime}\left(\breve{h}_{1}, \breve{h}_{2}\right) \\
= & \left(\frac{1}{\left|a_{1 i}\right|} \sum_{a_{k i} \in \breve{h}_{k}} \frac{1}{1-2^{1-q}}\left(\frac{\left(\theta_{1 \sigma(j)} / g\right)^{q}+\left(\theta_{2 \sigma(j)} / g\right)^{q}}{2}-\left(\frac{\theta_{1 \sigma(j)} / g+\theta_{2 \sigma(j)} / g}{2}\right)^{q}\right)^{p}\right)^{1 / p}+ \\
& \left(\frac{1}{\left|a_{1 i}\right|} \sum_{a_{k i} \in \breve{h}_{k}} \frac{1}{1-2^{1-q}}\left(\frac{\left(1-\theta_{1 \sigma(j)} / g\right)^{q}+\left(1-\theta_{2 \sigma(j)} / g\right)^{q}}{2}-\left(1-\frac{\theta_{1 \sigma(j)} / g+\theta_{2 \sigma(j)} / g}{2}\right)^{q}\right)^{p}\right)^{1 / p} \\
& +\left(\frac{1}{\left|a_{1 i}\right|\left|\operatorname{lh}\left(s_{\left(\theta_{k i}\right)}\right)\right|}\left(\mu_{i j}^{(k)}, v_{i j}^{(k)}\right) \in \operatorname{lh}\left(s_{\theta_{i j}}\right)\right. \\
& +\left(\frac{1}{1-2^{1-q}}\left(\frac{\left(\mu_{1 \sigma(j)}^{(k)}\right)^{q}+\left(\mu_{2 \sigma(j)}^{(k)}\right)^{q}}{2}-\left(\frac{\mu_{1 \sigma(j)}^{(k)}+\mu_{2 \sigma(j)}^{(k)}}{2}\right)^{q}\right)^{p}\right)^{1 / p} \\
& 2 \geqslant q>1, p \geqslant 1 .
\end{aligned}
$$

By using the concave-up function $f(x)=(1+$ $q x) \ln (1+q x)$, we can extend the cross-entropy measures $C E_{7}\left(\widetilde{\alpha}_{1}, \widetilde{\alpha}_{2}\right)$ and $C E_{8}\left(\widetilde{\alpha}_{1}, \widetilde{\alpha}_{2}\right)$ for intuitionistic hesitant fuzzy values to linguistic hesitant intuitionistic fuzzy environment to get cross-entropy measures $C E_{9}^{\prime}\left(\check{h}_{1}, \breve{h}_{2}\right)$ and $C E_{10}^{\prime}\left(\check{h}_{1}, \check{h}_{2}\right)$.

$$
\begin{aligned}
& C E_{9}^{\prime}\left(\breve{h}_{1}, \check{h}_{2}\right) \\
& =\frac{1}{T}\left(\operatorname { m a x } _ { a _ { k i } \in \breve { h } _ { k } } \left\{\frac{\left(1+q\left(\theta_{1 \sigma(i)} / g\right)\right) \ln \left(1+q\left(\theta_{1 \sigma(i)} / g\right)\right)+\left(1+q\left(\theta_{2 \sigma(i)} / g\right)\right) \ln \left(1+q\left(\theta_{2 \sigma(i)} / g\right)\right)}{2}-\right.\right. \\
& \left.\frac{2+q\left(\theta_{1 \sigma(i)} / g\right)+q\left(\theta_{2 \sigma(i)} / g\right)}{2} \ln \frac{2+q\left(\theta_{1 \sigma(i)} / g\right)+q\left(\theta_{2 \sigma(i)} / g\right)}{2}\right\}+ \\
& \max _{a_{k i} \in \breve{h}_{k}}\left\{\frac{\left(1+q\left(1-\theta_{1 \sigma(i)} / g\right)\right) \ln \left(1+q\left(1-\theta_{1 \sigma(i)} / g\right)\right)+\left(1+q\left(1-\theta_{2 \sigma(i)} / g\right)\right) \ln \left(1+q\left(1-\theta_{2 \sigma(i)} / g\right)\right)}{2}-\right. \\
& \left.\frac{2+q\left(1-\theta_{1 \sigma(i)} / g\right)+q\left(1-\theta_{2 \sigma(i)} / g\right)}{2} \ln \frac{2+q\left(1-\theta_{1 \sigma(i)} / g\right)+q\left(1-\theta_{2 \sigma(i)} / g\right)}{2}\right\}+ \\
& \max _{\left(\mu_{i j}^{(k)}, v_{i j}^{(k)}\right) \in \operatorname{lh}\left(s_{\theta_{i j}}\right)}\left\{\frac{\left(1+q \mu_{1 \sigma(j)}^{(k)}\right) \ln \left(1+q \mu_{1 \sigma(j)}^{(k)}\right)+\left(1+q \mu_{2 \sigma(j)}^{(k)}\right) \ln \left(1+q \mu_{2 \sigma(j)}^{(k)}\right)}{2}-\right. \\
& \left.\frac{2+q \mu_{1 \sigma(j)}^{(k)}+q \mu_{2 \sigma(j)}^{(k)}}{2} \ln \frac{2+q \mu_{1 \sigma(j)}^{(k)}+q \mu_{2 \sigma(j)}^{(k)}}{2}\right\}+ \\
& \max _{\left(\mu_{i j}^{(k)}, v_{i j}^{(k)}\right) \in \operatorname{lh}\left(s_{\left.\theta_{i j}\right)}\right.}\left\{\frac{\left(1+q v_{1 \sigma(j)}^{(k)}\right) \ln \left(1+q v_{1 \sigma(j)}^{(k)}\right)+\left(1+q v_{2 \sigma(j)}^{(k)}\right) \ln \left(1+q v_{2 \sigma(j)}^{(k)}\right)}{2}-\right. \\
& \left.\left.\frac{2+q v_{1 \sigma(j)}^{(k)}+q v_{2 \sigma(j)}^{(k)}}{2} \ln \frac{2+q v_{1 \sigma(j)}^{(k)}+q v_{2 \sigma(j)}^{(k)}}{2}\right\}\right), q>0 .
\end{aligned}
$$




$$
\begin{aligned}
& C E_{10}^{\prime}\left(\check{h}_{1}, \breve{h}_{2}\right) \\
& =\left(\frac { 1 } { | a _ { 1 i } | T } \sum _ { a _ { k i } \in \check { h } _ { k } } \left(\frac{\left(1+q\left(\theta_{1 \sigma(i)} / g\right)\right) \ln \left(1+q\left(\theta_{1 \sigma(i)} / g\right)\right)+\left(1+q\left(\theta_{2 \sigma(i)} / g\right)\right) \ln \left(1+q\left(\theta_{2 \sigma(i)} / g\right)\right)}{2}\right.\right. \\
& \left.\left.-\frac{2+q\left(\theta_{1 \sigma(i)} / g\right)+q\left(\theta_{2 \sigma(i)} / g\right)}{2} \ln \frac{2+q\left(\theta_{1 \sigma(i)} / g\right)+q\left(\theta_{2 \sigma(i)} / g\right)}{2}\right)^{p}\right)^{1 / p}+ \\
& \left(\frac { 1 } { | a _ { 1 i } | T } \sum _ { a _ { k i } \in \breve { h } _ { k } } \left(\frac{\left(1+q\left(1-\theta_{1 \sigma(i)} / g\right)\right) \ln \left(1+q\left(1-\theta_{1 \sigma(i)} / g\right)\right)+\left(1+q\left(1-\theta_{2 \sigma(i)} / g\right)\right) \ln \left(1+q\left(1-\theta_{2 \sigma(i)} / g\right)\right)}{2}\right.\right. \\
& \left.\left.-\frac{2+q\left(\theta_{1 \sigma(i)} / g\right)+q\left(1-\theta_{2 \sigma(i)} / g\right)}{2} \ln \frac{2+q\left(1-\theta_{1 \sigma(i)} / g\right)+q\left(1-\theta_{2 \sigma(i)} / g\right)}{2}\right)^{p}\right)^{1 / p}+ \\
& \left(\frac { 1 } { | a _ { 1 i } | | \operatorname { l h } ( s _ { \theta _ { k i } | } | T } \sum _ { ( \mu _ { i j } ^ { ( k ) } , v _ { i j } ^ { ( k ) } ) \in \operatorname { l h } ( s _ { \theta _ { i j } } ) } \left(\frac{\left(1+q \mu_{1 \sigma(j)}^{(k)}\right) \ln \left(1+q \mu_{1 \sigma(j)}^{(k)}\right)+\left(1+q \mu_{2 \sigma(j)}^{(k)}\right) \ln \left(1+q \mu_{2 \sigma(j)}^{(k)}\right)}{2}-\right.\right. \\
& \left.\left.\frac{2+q \mu_{1 \sigma(j)}^{(k)}+q \mu_{2 \sigma(j)}^{(k)}}{2} \ln \frac{2+q \mu_{1 \sigma(j)}^{(k)}+q \mu_{2 \sigma(j)}^{(k)}}{2}\right)^{p}\right)^{1 / p}+ \\
& \left(\frac { 1 } { | a _ { 1 i } | | \operatorname { l h } ( s _ { ( \theta _ { k i } ) } ) | T } \sum _ { ( \mu _ { i j } ^ { ( k ) } , v _ { i j } ^ { ( k ) } ) \in \operatorname { l h } ( s _ { \theta _ { i j } ) } } \left(\frac{\left(1+q v_{1 \sigma(j)}^{(k)}\right) \ln \left(1+q v_{1 \sigma(j)}^{(k)}\right)+\left(1+q v_{2 \sigma(j)}^{(k)}\right) \ln \left(1+q v_{2 \sigma(j)}^{(k)}\right)}{2}-\right.\right. \\
& \left.\left.\frac{2+q v_{1 \sigma(j)}^{(k)}+q v_{2 \sigma(j)}^{(k)}}{2} \ln \frac{2+q v_{1 \sigma(j)}^{(k)}+q v_{2 \sigma(j)}^{(k)}}{2}\right)^{p}\right)^{1 / p} \text {. }
\end{aligned}
$$

Here $q>0, p \geqslant 1$ and $T=(1+q) \ln (1+q)-(2+q)(\ln (2+q)-\ln 2)$.

Theorem 5. The measures defined in Eqs.(16)(25) are linguistic hesitant intuitionisic fuzzy crossentropy measures, which satisfy the conditions given in Definition 3.1.

Proof. We prove the Eq.(16) and other equations

$$
\begin{aligned}
C E_{1}^{\prime}\left(\breve{h}_{1}, \breve{h}_{2}\right)= & \max _{a_{k i} \in \breve{h}_{k}}\left\{\frac{\theta_{1 \sigma(i)}}{g} \log _{2} \frac{2 \theta_{1 \sigma(i)}}{\theta_{1 \sigma(i)}+\theta_{2 \sigma(i)}}+\left(1-\frac{\theta_{1 \sigma(i)}}{g}\right) \log _{2} \frac{2\left(g-\theta_{1 \sigma(i)}\right)}{2 g-\theta_{1 \sigma(i)}-\theta_{2 \sigma(i)}}\right\}+ \\
& \max _{\left(\mu_{i j}^{(k)}, v_{i j}^{(k)}\right) \in \ln \left(s_{\theta_{i j}}\right)}\left\{\mu_{1 \sigma(j)}^{(k)} \log _{2} \frac{2 \mu_{1 \sigma(j)}^{(k)}}{\mu_{1 \sigma(j)}^{(k)}+\mu_{2 \sigma(j)}^{(k)}}+v_{1 \sigma(j)}^{(k)} \log _{2} \frac{2 v_{1 \sigma(j)}^{(k)}}{v_{1 \sigma(j)}^{(k)}+v_{2 \sigma(j)}^{(k)}}\right\} \\
= & \max _{a_{k i} \in \breve{h}_{k}}\left\{\frac{\theta_{1 \sigma(i)}}{g} \log _{2} \frac{2 \theta_{1 \sigma(i)}}{\theta_{1 \sigma(i)}+\theta_{1 \sigma(i)}}+\left(1-\frac{\theta_{1 \sigma(i)}}{g}\right) \log _{2} \frac{2\left(g-\theta_{1 \sigma(i)}\right)}{2 g-\theta_{1 \sigma(i)}-\theta_{1 \sigma(i)}}\right\}+ \\
& \left(\mu_{i j}^{(k)}, v_{i j}^{(k)}\right) \in \operatorname{lh}\left(s_{\left.\theta_{i j}\right)}\left\{\mu_{1 \sigma(j)}^{(k)} \log _{2} \frac{2 \mu_{1 \sigma(j)}^{(k)}}{\mu_{1 \sigma(j)}^{(k)}+\mu_{1 \sigma(j)}^{(k)}}+v_{1 \sigma(j)}^{(k)} \log _{2} \frac{2 v_{1 \sigma(j)}^{(k)}}{v_{1 \sigma(j)}^{(k)}+v_{1 \sigma(j)}^{(k)}}\right\}\right. \\
= & \max _{a_{k i} \in \check{h}_{k}}\{0\}+\max _{\left(\mu_{i j}^{(k)}, v_{i j}^{(k)}\right) \in \operatorname{lh}\left(s_{\left.\theta_{i j}\right)}\{0\}=0 .\right.}\{0\}
\end{aligned}
$$

$$
\begin{aligned}
C E_{1}^{\prime}\left(\breve{h}_{1}^{c}, \breve{h}_{2}^{c}\right)= & \max _{a_{k i}^{c} \in \breve{h}_{k}^{c}}\left\{\frac{g-\theta_{1 \sigma(i)}}{g} \log _{2} \frac{2\left(g-\theta_{1 \sigma(i)}\right)}{\left(g-\theta_{1 \sigma(i)}\right)+\left(g-\theta_{2 \sigma(i)}\right)}+\left(1-\frac{g-\theta_{1 \sigma(i)}}{g}\right) \log _{2} \frac{2\left(g-\left(g-\theta_{1 \sigma(i)}\right)\right)}{2 g-\left(g-\theta_{1 \sigma(i)}\right)-\left(g-\theta_{2 \sigma(i)}\right)}\right\} \\
& +\max _{\left(v_{i j}^{(k)}, \mu_{i j}^{(k)}\right) \in \operatorname{lh}\left(s_{\left.\theta_{i j}\right)^{c}}\right.}\left\{v_{1 \sigma(j)}^{(k)} \log _{2} \frac{2 v_{1 \sigma(j)}^{(k)}}{v_{1 \sigma(j)}^{(k)}+v_{2 \sigma(j)}^{(k)}}+\mu_{1 \sigma(j)}^{(k)} \log _{2} \frac{2 \mu_{1 \sigma(j)}^{(k)}}{\mu_{1 \sigma(j)}^{(k)}+\mu_{2 \sigma(j)}^{(k)}}\right\},
\end{aligned}
$$




$$
\begin{aligned}
C E_{1}^{\prime}\left(\check{h}_{1}^{c}, \check{h}_{2}^{c}\right)= & \max _{a_{k i} \in \breve{h}_{k}}\left\{\left(1-\frac{\theta_{1 \sigma(i)}}{g}\right) \log _{2} \frac{2\left(g-\theta_{1 \sigma(i)}\right)}{2 g-\theta_{1 \sigma(i)}-\theta_{1 \sigma(i)}}+\frac{\theta_{1 \sigma(i)}}{g} \log _{2} \frac{2 \theta_{1 \sigma(i)}}{\theta_{1 \sigma(i)}+\theta_{1 \sigma(i)}}\right\}+ \\
& \max _{\left(\mu_{i j}^{(k)}, v_{i j}^{(k)}\right) \in \ln \left(s_{\left.\theta_{i j}\right)}\right)}\left\{\mu_{1 \sigma(j)}^{(k)} \log _{2} \frac{2 \mu_{1 \sigma(j)}^{(k)}}{\mu_{1 \sigma(j)}^{(k)}+\mu_{1 \sigma(j)}^{(k)}}+v_{1 \sigma(j)}^{(k)} \log _{2} \frac{2 v_{1 \sigma(j)}^{(k)}}{v_{1 \sigma(j)}^{(k)}+v_{1 \sigma(j)}^{(k)}}\right\} \\
= & C E_{1}^{\prime}\left(\breve{h}_{1}, \breve{h}_{2}\right) .
\end{aligned}
$$

The proof is thus complete.

Several linguistic hesitant intuitionisic fuzzy cross-entropy measures have been introduced in this paper, each cross-entropy measure has its own characteristics and emphasis. Decision makers can select the cross-entropy measure according to the real needs and his preference.

The proposed cross-entropy measures are the degrees of discrimination of $\breve{h}_{1}$ from $\breve{h}_{2}$. However, $C E_{i}^{\prime}\left(\breve{h}_{1}, \breve{h}_{2}\right)(i=1,2, \ldots, 10)$ are not symmetric with respect to their arguments. Symmetric form of cross-entropy measures for LHIFEs can be got by modifying the Eqs. (16)-(25) as follows:

$$
C E_{i}^{*}\left(\breve{h}_{1}, \breve{h}_{2}\right)=C E_{i}^{\prime}\left(\breve{h}_{1}, \breve{h}_{2}\right)+C E_{i}^{\prime}\left(\breve{h}_{2}, \breve{h}_{1}\right) .
$$

Example: Let $\breve{h}_{1}=\left\{\left(s_{5},(0.7,0.2),(0.6,0.3)\right)\right.$, $\left.\left(s_{6},(0.6,0.2),(0.5,0.4)\right),\left(s_{7},(0.5,0.3),(0.6,0.4)\right)\right\}$, $\breve{h}_{2}=\left\{\left(s_{6},(0.8,0.1),(0.7,0.3)\right),\left(s_{7},(0.6,0.2),(0.5\right.\right.$, $\left.0.3)),\left(s_{8},(0.5,0.2),(0.5,0.3)\right)\right\}$ be two patterns and $\breve{h}=\left\{\left(s_{4},(0.7,0.1),(0.6,0.2)\right),\left(s_{5},(0.7,0.2)\right.\right.$, $\left.(0.6,0.3)),\left(s_{6},(0.6,0.2),(0.6,0.3)\right)\right\}$ be a sample. $p=q=2$, then the following results can be got:

$$
C E_{1}^{*}\left(\breve{h}_{1}, \breve{h}\right)=0.2636, \quad C E_{1}^{*}\left(\breve{h}_{2}, \breve{h}\right)=0.3359,
$$
$C E_{2}^{*}\left(\breve{h}_{1}, \breve{h}\right)=0.0480, \quad C E_{2}^{*}\left(\breve{h}_{2}, \breve{h}\right)=0.0978$, $C E_{3}^{*}\left(\breve{h}_{1}, \breve{h}\right)=0.3152, \quad C E_{3}^{*}\left(\breve{h}_{2}, \breve{h}\right)=0.4811$, $C E_{4}^{*}\left(\breve{h}_{1}, \breve{h}\right)=0.0571, \quad C E_{4}^{*}\left(\breve{h}_{2}, \breve{h}\right)=0.1227$, $C E_{5}^{*}\left(\breve{h}_{1}, \breve{h}\right)=0.6137, \quad C E_{5}^{*}\left(\breve{h}_{2}, \breve{h}\right)=0.8592$, $C E_{6}^{*}\left(\breve{h}_{1}, \breve{h}\right)=0.5824, \quad C E_{6}^{*}\left(\breve{h}_{2}, \breve{h}\right)=0.7987$, $C E_{7}^{*}\left(\breve{h}_{1}, \breve{h}\right)=0.1867, \quad C E_{7}^{*}\left(\breve{h}_{2}, \breve{h}\right)=0.2969$, $C E_{8}^{*}\left(\breve{h}_{1}, \breve{h}\right)=0.0394, \quad C E_{8}^{*}\left(\breve{h}_{2}, \breve{h}\right)=0.0810$, $C E_{9}^{*}\left(\breve{h}_{1}, \breve{h}\right)=0.0840, \quad C E_{9}^{*}\left(\breve{h}_{2}, \breve{h}\right)=0.1322$, $C E_{10}^{*}\left(\breve{h}_{1}, \breve{h}\right)=0.0432, C E_{10}^{*}\left(\breve{h}_{2}, \breve{h}\right)=0.0830$.

From the above results we can see that the sample $\breve{h}$ belongs to the pattern $\breve{h}_{1}$ by using all the above cross-entropy measures.

\section{New MADM methods based on the cross-entropy measures of LHIFEs}

Considering a multiple attribute decision making problem, let $A=\left\{A_{1}, A_{2}, \ldots, A_{m}\right\}$ be a finite alternative set and $C=\left\{C_{1}, C_{2}, \ldots, C_{n}\right\}$ be a finite attribute set. The decision makers evaluate alternatives with respect to attributes with linguistic terms and intuitionisic fuzzy memberships. If two or more decision makers gave the same LIFE in evaluating the same alternative with respect to some attribute, it is counted only once. The linguistic hesitant intuitionistic fuzzy decision matrix can be got as $\widetilde{D}=$ $\left(\check{h}_{i j}\right)_{m \times n}$, where $\check{h}_{i j}=\left\{\left(s_{\theta_{i j}}, \operatorname{lh}\left(s_{\theta_{i j}}\right) \mid\left(\mu_{i j}^{(t)}, v_{i j}^{(t)}\right) \in\right.\right.$ $\left.\operatorname{lh}\left(s_{\theta_{i j}}\right), t=1,2, \ldots, l_{i j}\right\}$ is LHIFE. $\left\{s_{\theta_{i j}}\right\}$ is the linguistic terms given by experts to evaluate alternative $A_{i}$ with respect to the attribute $C_{j}, \mu_{i j}^{(t)}$ indicates the degree of linguistic term $s_{\theta_{i j}}$ satisfying the attribute $C_{j}$ and $v_{i j}^{(t)}$ indicates the degree of linguistic term $s_{\theta_{i j}}$ dissatisfying the attribute $C_{j}$, such that $\mu_{i j}^{(t)}, v_{i j}^{(t)} \in[0,1]$ and $\mu_{i j}^{(t)}+v_{i j}^{(t)} \leqslant 1$.

Different LHIFEs may have different number of LIFEs and different LIFEs may have different number of intuitionistic fuzzy memberships. In order to define cross-entropy measures more accurately, we extend LHIFEs according to the risk attitudes of decision makers until all LHIFEs have the same number of LIFEs and all LIFEs have the same number of intuitionistic fuzzy memberships. If the decision maker is risk-seeking, the largest LIFE and largest intuitionistic fuzzy membership can be added; if the decision maker is risk-averse, the smallest LIFE and smallest intuitionistic fuzzy membership can be added; if the decision maker is risk-neutral, the average LIFE and average intuitionistic fuzzy membership can be added.

In some decision making process, information for attribute weight is partly known or unknown 
completely due to decision time pressure, decision makers' lack of knowledge and expertise, complicated decision problems, etc. Generally, partly known attribute information can be expressed as a subset of the following relations: a weak ranking: $\left\{w_{i} \geqslant w_{j}\right\}, i \neq j$; a strict ranking: $\left\{w_{i}-w_{j} \geqslant\right.$ $\left.\varepsilon_{i}(>0)\right\}, i \neq j$; a ranking with multiples: $\left\{w_{i} \geqslant\right.$ $\left.\alpha_{i} w_{j}\right\}, 0 \leqslant \alpha_{i} \leqslant 1, i \neq j$; an interval form: $\left\{\beta_{j} \leqslant\right.$ $\left.w_{j} \leqslant \beta_{j}+\varepsilon_{j}\right\}, 0 \leqslant \beta_{j}<\beta_{j}+\varepsilon_{j} \leqslant 1$; a ranking of differences: $\left\{w_{i}-w_{j} \geqslant w_{k}-w_{l}\right\}$, for $i \neq j \neq k \neq l$. For a specific decision problem, attribute weight information can be described as a subset of the above relationships. The corresponding attribute weight information set can be denoted as $H$. According to information theory, an attribute should be assigned a larger weight if its evaluation values have obvious differences since it plays an important role in the priority procedure. Otherwise, it should be assigned a smaller weight ${ }^{51}$. Since several linguistic hesitant intuitionistic fuzzy cross-entropy measures have been developed, we only choose one crossentropy measure for the convenience of calculation and analysis and we can calculate similarly if other cross-entropy measures are chosen. Then the deviation value $d_{j}$ of attribute $C_{j}$ can be calculated as

$$
d_{j}=\sum_{i=1}^{m} \sum_{k=1}^{m} C E^{*}\left(\check{h}_{i j}, \check{h}_{k j}\right) .
$$

The weighted deviation value can be calculated as

$$
\begin{aligned}
d & =\sum_{j=1}^{n} w_{j} d_{j} \\
& =\sum_{j=1}^{n} w_{j}\left(\sum_{i=1}^{m} \sum_{k=1}^{m} C E^{*}\left(\check{h}_{i j}, \breve{h}_{k j}\right)\right) \\
& =\sum_{j=1}^{n} \sum_{i=1}^{m} \sum_{k=1}^{m} w_{j} C E^{*}\left(\check{h}_{i j}, \breve{h}_{k j}\right) .
\end{aligned}
$$

A reasonable weight vector $w=\left(w_{1}, w_{2}, \ldots, w_{n}\right)$ should make the weighted deviation value as large as possible to differentiate the problem characteristics more effectively ${ }^{51}$. Thus we set up the following programming model to determine optimal attribute weights if attribute weights are partly known.

$$
\begin{array}{cl}
\text { (M-1) } & \sum_{j=1}^{n} \sum_{i=1}^{m} \sum_{k=1}^{m} w_{j} C E^{*}\left(\breve{h}_{i j}, \breve{h}_{k j}\right) \\
\text { s.t. } & w \in H, \\
& w_{j} \geqslant 0, j=1,2, \ldots, n \\
& w_{1}+w_{2}+\ldots+w_{n}=1 .
\end{array}
$$

The model (M-1) is a linear programming model, which can be solved easily by using many existing methods.

If information for attribute weights is unknown completely, we set up the following model

$$
\begin{array}{cl}
(\mathrm{M}-2) & \sum_{j=1}^{n} \sum_{i=1}^{m} \sum_{k=1}^{m} w_{j} C E^{*}\left(\breve{h}_{i j}, \breve{h}_{k j}\right) \\
\text { s.t. } & \sum_{j=1}^{n} w_{j}^{2}=1, \\
& w_{j} \geqslant 0, j=1,2, \ldots, n .
\end{array}
$$

In order to solve model (M-2), we construct the following Lagrange function:

$L(W, \lambda)=\sum_{j=1}^{n} \sum_{i=1}^{m} \sum_{k=1}^{m} w_{j} C E^{*}\left(\check{h}_{i j}, \breve{h}_{k j}\right)+\frac{\lambda}{2}\left(\sum_{j=1}^{n} w_{j}^{2}-1\right)$,

where $\lambda$ is the Lagrange multiplier. Calculate the differentiation of Eq. (29) with respect to $w_{j}(j=$ $1,2, \ldots, n)$ and $\lambda$, and set these partial derivatives equal to zeros to get

$$
\left\{\begin{array}{l}
\frac{\partial L}{\partial w_{j}}=\sum_{i=1}^{m} \sum_{k=1}^{m} C E^{*}\left(\breve{h}_{i j}, \breve{h}_{k j}\right)+\lambda w_{j}=0, \\
\frac{\partial L}{\partial \lambda}=\sum_{j=1}^{n} w_{j}^{2}-1=0 .
\end{array}\right.
$$

By solving Eq.(30), we can get the formula for calculating attribute weights as

$$
w_{j}=\frac{\sum_{i=1}^{m} \sum_{k=1}^{m} C E^{*}\left(\check{h}_{i j}, \check{h}_{k j}\right)}{\sqrt{\sum_{j=1}^{n}\left(\sum_{i=1}^{m} \sum_{k=1}^{m} C E^{*}\left(\check{h}_{i j}, \check{h}_{k j}\right)\right)^{2}}}, j=1,2, \ldots, n .
$$

Normalize the attribute weights to get

$$
w_{j}=\frac{\sum_{i=1}^{m} \sum_{k=1}^{m} C E^{*}\left(\breve{h}_{i j}, \breve{h}_{k j}\right)}{\sum_{j=1}^{n} \sum_{i=1}^{m} \sum_{k=1}^{m} C E^{*}\left(\check{h}_{i j}, \breve{h}_{k j}\right)}, j=1,2, \ldots, n .
$$

Based on the above analysis, we can present an effective approach to solve the multiple attribute decision making problem with linguistic hesitant intuitionistic fuzzy information.

\section{Algorithm I}

Step 1. Construct the decision matrix $\widetilde{D}=$ $\left(\breve{h}_{i j}\right)_{m \times n}$. Multiple decision makers evaluate the alternatives with respect to attributes with linguistic terms and intuitionistic fuzzy memberships. Then linguistic hesitant intuitionistic fuzzy elements are formed as $\breve{h}_{i j}$.

Step 2. If attribute weights are known completely, go to Step 3 directly. In order to calculate 
the cross-entropy measure more accurately, we extend the decision matrix according to the risk attitude of decision makers and the decision matrix $\widetilde{D}^{\prime}=\left(\breve{h}_{i j}^{\prime}\right)_{m \times n}$ can be got. If attribute weights are known partly, we can solve model (M-1) to obtain them; if attribute weights are completely unknown, we can calculate them by using Eq.(32).

Step 3. Calculate the collective evaluation values of alternatives by using the decision matrix $\widetilde{D}=$ $\left(\breve{h}_{i j}\right)_{m \times n}$. We can calculate by using the LHIFWA operator, the LHIFWG operator or the GLHIFWA operator.

Step 4. Calculate the scores $S\left(\check{h}_{i}\right) \quad(i=$ $1,2, \ldots, m)$ and the accuracy degrees $A\left(\breve{h}_{i}\right) \quad(i=$ $1,2, \ldots, m)$ of alternatives' collective evaluation values $\breve{h}_{i}(i=1,2, \ldots, m)$ by using the score function and accuracy function.

$$
\begin{aligned}
& S\left(\check{h}_{i}\right) \\
= & \frac{1}{\mid \breve{h_{i} \mid}}\left(\sum \frac{\theta_{i}}{g \mid l\left(s_{\theta_{i}}\right)} \sum_{\left(\alpha_{i}^{(k)}, \beta_{i}^{(k)}\right) \in \operatorname{lh}\left(s_{\theta_{i}}\right)}\left(\mu_{i}^{(k)}-v_{i}^{(k)}\right)\right), \\
= & A\left(\check{h}_{i}\right) \\
= & \frac{1}{\left|\breve{h}_{i}\right|}\left(\sum \frac{\theta_{i}}{g\left|l\left(s_{\theta_{i}}\right)\right|} \sum_{\left(\alpha_{i}^{(k)}, \beta_{i}^{(k)}\right) \in \operatorname{lh}\left(s_{\theta_{i}}\right)}\left(\mu_{i}^{(k)}+v_{i}^{(k)}\right)\right),
\end{aligned}
$$

where $\left|\breve{h}_{i}\right|$ and $\left|\operatorname{lh}\left(s_{\theta(i)}\right)\right|$ are the cardinalities of $\check{h}_{i}$ and $\operatorname{lh}\left(s_{\theta_{i}}\right)$, respectively. $\check{h}_{i}=\left\{\left(s_{\theta_{i}}, \operatorname{lh}\left(s_{\theta_{i}}\right)\right)\right\}$, $\operatorname{lh}\left(s_{\theta_{i}}\right)=\left\{\left(\mu_{i}^{(k)}, v_{i}^{(k)}\right)\right\}$.

Step 5. Rank $\breve{h}_{i}$ according the method given in Definition 2.6 and rank alternatives accordingly.

In LHIFEs, intuitionistic fuzzy memberships and nonmemberships have been considered besides linguistic evaluation values. Hence, aggregation of linguistic hesitant intuitionistic fuzzy information is more complex than linguistic hesitant fuzzy sets. By calculating the number of basic operations at each step, we can get the worst-case time complexity of our algorithm is $O\left(m^{2} n l t\right)$, where $m$ is the number of alternatives, $n$ is the number of attributes, $l$ is the largest number of LIFEs in LHIFEs, $t$ is the largest number of intuitionistic fuzzy values in LIFEs. Then the complexity of algorithm can tell us the new algorithm is an efficient and practical polynomial-time algorithm for solving multiple attribute decision making problems.

TOPSIS method was developed by Hwang and
Yong $^{52}$, which is based on the principle that the optimal alternative should have the shortest distance from the positive ideal solution and at the same time have the farthest distance from the negative ideal solution. From the risk viewpoint, decision makers are risk-averse since they choose the alternative which is not only making as much profit as possible, but also avoiding as much risk as possible. In the following, we present a new ranking method based on the cross-entropy measures and the idea of TOPSIS.

\section{Algorithm II}

Step 1. As for Algorithm I.

Step 2. As for Algorithm I.

Step 3. Determine the linguistic hesitant intuitionistic fuzzy positive-ideal solution (LHIFPIS) as

$$
\check{h}^{+}=\left\{\left(s_{g},\{(1,0), \ldots,(1,0)\}\right)\right\},
$$

and linguistic hesitant intuitionistic fuzzy negativeideal solution (LHIFNIS) as

$$
\check{h}^{-}=\left\{\left(s_{1},\{(0,1), \ldots,(1,0)\}\right)\right\} .
$$

Each $\check{h}^{+}$and $\check{h}^{-}$have the same number of LHIFEs and LIFEs as $\breve{h}_{i j}^{\prime}$.

Step 4. Calculate the symmetric cross-entropy of $\check{h}_{i j}^{\prime}$ from $\breve{h}^{+}$and $\breve{h}^{-}$as $G_{i j}^{+}$, $G_{i j}^{-}$, respectively.

$$
\begin{aligned}
& G_{i j}^{+}=C_{2}^{*}\left(\check{h}_{i j}^{\prime}, \check{h}^{+}\right)=C_{2}\left(\check{h}_{i j}^{\prime}, \check{h}^{+}\right)+C_{2}\left(\check{h}^{+}, \check{h}_{i j}^{\prime}\right), \\
& G_{i j}^{-}=C_{2}^{*}\left(\check{h}_{i j}^{\prime}, \check{h}^{-}\right)=C_{2}\left(\check{h}_{i j}^{\prime}, \check{h}^{-}\right)+C_{2}\left(\check{h}^{-}, \check{h}_{i j}^{\prime}\right) .
\end{aligned}
$$

Step 4. Determine the relative closeness by using the following equation

$$
G_{i j}=\frac{G_{i j}^{-}}{G_{i j}^{+}+G_{i j}^{-}}, i=1,2, \ldots, m, j=1,2, \ldots, n .
$$

Then the weighted relative closeness coefficients of alternatives can be calculated as follows

$$
G_{i}=\sum_{j=1}^{n} w_{j} G_{i j}, i=1,2, \ldots, m .
$$

Step 5. Rank alternatives according to the ranking of $G_{i}(i=1,2, \ldots, m)$. The larger the $G_{i}$, the better the alternative $A_{i}$. 


\section{Numerical example}

A numerical example adapted from Chen and Yang $^{53}$ is presented to illustrate efficiency and practical advantages of the proposed procedure.

Suppose that there is an architecture company wanting to select a company to supply an important material, such as cement. Experts from different departments have been invited and they mainly consider the following four attributes: $C_{1}-$ the price of product, $C_{2}-$ the quality of product, $C_{3}-$ delivery time, $C_{4}$-risk. After pre-evaluation, there are still five alternatives $A_{i}(i=1,2, \ldots, 5)$ left for further evaluation. We use the new algorithms to rank alternatives.

Step 1. The experts evaluate alternatives $A_{i}(i=1,2, \ldots, 5)$ with respect to attributes $C_{j}(j=$ $1,2, \ldots, 4)$ with linguistic terms and intuitionistic fuzzy memberships. The decision matrix is formed as $\widetilde{D}=\left(\breve{h}_{i j}\right)_{4 \times 5}$ in Table 1 .

Step 2. Assume attribute weights are known completely as $w_{1}=(0.15,0.20,0.30,0.35)$.

Step 3. Calculate alternatives' collective evaluation values. For example, we calculate $\breve{h}_{1}$ by using the LHIFWA operator as follows

$$
\begin{aligned}
\check{h}_{1}= & \left\{\left(s_{5.15},\{(0.6206,0.1803),(0.6418,\right.\right. \\
& 0.1803)\}),\left(s_{5.30},\{(0.6331,0.1726),\right. \\
& (0.6536,0.1726)\}),\left(s_{5.45},\{(0.5864,\right. \\
& 0.2219),(0.6095,0.2219)\}),\left(s_{5.60},\right. \\
& \{(0.6000,0.2125),(0.6224,0.2125)\})\} .
\end{aligned}
$$

Similarly, we can calculate other $\breve{h}_{i}(i=2,3, \ldots, 5)$.

Step 4. Calculate scores of collective evaluation values $\breve{h}_{i}(i=1,2, \ldots, 5)$ to get $S\left(\check{h}_{1}\right)=$ $0.2527, S\left(\breve{h}_{2}\right)=0.2493, S\left(\breve{h}_{3}\right)=0.2193, S\left(\breve{h}_{4}\right)=$ $0.2505, S\left(\breve{h}_{5}\right)=0.2601$. Rank $S\left(\breve{h}_{i}\right)(i=1,2, \ldots, 5)$ to get

$$
S\left(\breve{h}_{5}\right)>S\left(\check{h}_{1}\right)>S\left(\check{h}_{4}\right)>S\left(\check{h}_{2}\right)>S\left(\breve{h}_{3}\right) .
$$

Step 5. Rank $\breve{h}_{i}$ according to the ranking of $S\left(\breve{h}_{i}\right)$ to get

$$
\check{h}_{5}>\check{h}_{1}>\check{h}_{4}>\check{h}_{2}>\breve{h}_{3} \text {. }
$$

Then alternatives can be ranked accordingly as

$$
A_{5} \succ A_{1} \succ A_{4} \succ A_{2} \succ A_{3} .
$$

The optimal alternative is $A_{5}$.
We can use the LHIFWG operator or the

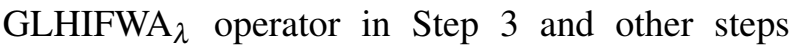
are the same as above. Then results can be obtained as shown in Table 3. If attribute weights are partly known or completely unknown, we need to calculate them first. Assume decision makers are risk-averse, then the smallest intuitionistic fuzzy value and LIFE can be added to extend the decision matrix as $\widetilde{D}^{\prime}$, which is shown in Table 2 . Though several cross-entropy measures have been introduced in this paper, we only choose one to calculate attribute weights for space limit. Here we use cross-entropy measures $C E_{2}^{*}$ and similar results can be got if other cross-entropy measures are used. If attribute weights are known partly, we can set up the following Model (M-3) to determine them as $w_{2}=(0.2782,0.3062,0.2279,0.1877)$. If attribute weights are unknown completely, we determine them by using Eq.(33) to get $w_{3}=$ $(0.30,0.15,0.35,0.20)$. Other steps can be calculated similarly as that of completely known attribute weights method above and results are shown in Table 3 . If attribute weight vector $w_{2}$ is used, $A_{2}$ becomes the optimal alternative and $A_{5}$ becomes the sub-optimal alternative in most case. For $w_{3}, A_{1}$ becomes the optimal alternative and $A_{5}$ becomes the sub-optimal alternative.

$$
\begin{array}{ll}
\text { (M-3) } & 1.9957 w_{1}+2.1970 w_{2}+1.6348 w_{3} \\
& +1.3467 w_{4} \\
\text { s.t. } & 0.15 \leqslant w_{1} \leqslant 0.30,0.10 \leqslant w_{2} \leqslant 0.25, \\
& 0.20 \leqslant w_{3} \leqslant 0.35,0.10 \leqslant w_{4} \leqslant 020 \\
& 2 w_{2} \leqslant w_{3}, w_{1}+w_{2}+\ldots+w_{4}=1 .
\end{array}
$$

If Algorithm II is used to rank alternatives, the first two steps are the same as that of Algorithm I. In Step 3, we determine the LHIFPIS $\breve{h}^{+}$and LHIFNIS $\breve{h}^{-}$as $\breve{h}^{+}=\left\{\check{a}^{+}\right\}, \breve{a}^{+}=\left(s_{9},\{(1,0),(1,0)\}\right), \check{h}^{-}=$ $\left\{\check{a}^{-}\right\}, \breve{a}^{-}=\left(s_{1},\{(0,1),(0,1)\}\right)$. In Step 4, we calculate the symmetric cross-entropy of $\breve{h}_{i j}$ from $\breve{h}^{+}$and $\check{h}^{-}$. For example, we calculate $G_{i j}^{+}$and $G_{i j}^{-}$by using Eqs.(35)-(36) if $C E_{2}^{*}$ is chosen. The relative closeness coefficients can be calculated by using Eq. (37). For completely known attribute weight vector $w_{1}=$ $(0.15,0.20,0.30,0.35)$, the weighted relative closeness coefficients can be calculated by using Eq.(38) as $G_{1}=0.5588, G_{2}=0.5414, G_{3}=0.4701, G_{4}=$ 
Table 1: Decision matrix $\widetilde{D}$.

\begin{tabular}{lll}
\hline & $C_{1}$ & $C_{2}$ \\
\hline$A_{1}$ & $\left\{\left(s_{6},(0.5,0.4)\right),\left(s_{7},(0.6,0.3)\right)\right\}$ & $\left\{\left(s_{2},(0.6,0.2),(0.7,0.2)\right)\right\}$ \\
$A_{2}$ & $\left\{\left(s_{3},(0.5,0.2),(0.5,0.3)\right)\right\}$ & $\left\{\left(s_{7},(0.7,0.1)\right),\left(s_{8},(0.8,0.2)\right)\right\}$ \\
$A_{3}$ & $\left\{\left(s_{4},(0.6,0.1)\right)\right\}$ & $\left\{\left(s_{5},(0.7,0.3),(0.5,0.4)\right)\right\}$ \\
$A_{4}$ & $\left\{\left(s_{2},(0.6,0.2),(0.5,0.3)\right)\right\}$ & $\left\{\left(s_{6},(0.8,0.2)\right)\right\}$ \\
$A_{5}$ & $\left\{\left(s_{5},(0.7,0.3)\right),\left(s_{6},(0.6,0.2)\right)\right\}$ & $\left\{\left(s_{3},(0.5,0.2),(0.6,0.3)\right)\right\}$ \\
\hline & $C_{3}$ & $C_{4}$ \\
\hline$A_{1}$ & $\left\{\left(s_{7},(0.7,0.1)\right),\left(s_{8},(0.6,0.2)\right)\right\}$ & $\left\{\left(s_{5},(0.6,0.2)\right)\right\}$ \\
$A_{2}$ & $\left\{\left(s_{6},(0.6,0.3),(0.7,0.2)\right)\right\}$ & $\left\{\left(s_{4},(0.7,0.2)\right),\left(s_{5},(0.5,0.3),(0.6,0.4)\right)\right\}$ \\
$A_{3}$ & $\left\{\left(s_{2},(0.5,0.4)\right),\left(s_{3},(0.5,0.2)\right)\right\}$ & $\left\{\left(s_{8},(0.6,0.1),(0.7,0.2)\right)\right\}$ \\
$A_{4}$ & $\left\{\left(s_{3},(0.7,0.2),(0.6,0.3)\right)\right\}$ & $\left\{\left(s_{6},(0.6,0.2)\right),\left(s_{7},(0.7,0.1)\right)\right\}$ \\
$A_{5}$ & $\left\{\left(s_{7},(0.8,0.2)\right),\left(s_{8},(0.6,0.3)\right)\right\}$ & $\left\{\left(s_{4},(0.5,0.2)\right)\right\}$ \\
\hline
\end{tabular}

Table 2: Extended decision matrix $\widetilde{D}^{\prime}$.

\begin{tabular}{llll}
\hline & $C_{1}$ & $C_{2}$ \\
\hline$A_{1}$ & $\left\{\left(s_{6},(0.5,0.4),(0.5,0.4)\right),\left(s_{7},(0.6,0.3),(0.5,0.4)\right)\right\}$ & $\left\{\left(s_{2},(0.6,0.2),(0.7,0.2)\right),\left(s_{2},(0.5,0.4),(0.5,0.4)\right)\right\}$ \\
$A_{2}$ & $\left\{\left(s_{3},(0.5,0.2),(0.5,0.3)\right),\left(s_{2},(0.5,0.4),(0.5,0.4)\right)\right\}$ & $\left\{\left(s_{7},(0.7,0.1),(0.5,0.4)\right),\left(s_{8},(0.8,0.2),(0.5,0.4)\right)\right\}$ \\
$A_{3}$ & $\left\{\left(s_{4},(0.6,0.1),(0.5,0.4),\left(s_{2},(0.5,0.4),(0.5,0.4)\right)\right\}\right.$ & $\left\{\left(s_{5},(0.7,0.3),(0.5,0.4)\right),\left(s_{2},(0.5,0.4),(0.5,0.4)\right)\right\}$ \\
$A_{4}$ & $\left\{\left(s_{2},(0.6,0.2),(0.5,0.3)\right),\left(s_{2},(0.5,0.4),(0.5,0.4)\right)\right\}$ & $\left\{\left(s_{6},(0.8,0.2),(0.5,0.4)\right),\left(s_{2},(0.5,0.4),(0.5,0.4)\right)\right\}$ \\
$A_{5}$ & $\left\{\left(s_{5},(0.7,0.3),(0.5,0.4)\right),\left(s_{6},(0.6,0.2),(0.5,0.4)\right)\right\}$ & $\left\{\left(s_{3},(0.5,0.2),(0.6,0.3)\right),\left(s_{2},(0.5,0.4),(0.5,0.4)\right)\right\}$ \\
\hline & $C_{3}$ & $C_{4}$ \\
\hline$A_{1}$ & $\left\{\left(s_{7},(0.7,0.1),(0.5,0.4)\right),\left(s_{8},(0.6,0.2),(0.5,0.4)\right)\right\}$ & $\left\{\left(s_{5},(0.6,0.2),(0.5,0.4)\right),\left(s_{2},(0.5,0.4),(0.5,0.4)\right)\right\}$ \\
$A_{2}$ & $\left\{\left(s_{6},(0.6,0.3),(0.7,0.2)\right),\left(s_{2},(0.5,0.4),(0.5,0.4)\right)\right\}$ & $\left\{\left(s_{4},(0.7,0.2),(0.5,0.4)\right),\left(s_{5},(0.5,0.3),(0.6,0.4)\right)\right\}$ \\
$A_{3}$ & $\left\{\left(s_{2},(0.5,0.4),(0.5,0.4)\right),\left(s_{3},(0.5,0.2),(0.5,0.4)\right)\right\}$ & $\left\{\left(s_{8},(0.6,0.1),(0.7,0.2)\right),\left(s_{2},(0.5,0.4),(0.5,0.4)\right)\right\}$ \\
$A_{4}$ & $\left\{\left(s_{3},(0.7,0.2),(0.6,0.3)\right),\left(s_{2},(0.5,0.4),(0.5,0.4)\right)\right\}$ & $\left\{\left(s_{6},(0.6,0.2),(0.5,0.4)\right),\left(s_{7},(0.7,0.1),(0.5,0.4)\right)\right\}$ \\
$A_{5}$ & $\left\{\left(s_{7},(0.8,0.2),(0.5,0.4)\right),\left(s_{8},(0.6,0.3),(0.5,0.4)\right)\right\}$ & $\left\{\left(s_{4},(0.5,0.2),(0.5,0.4)\right),\left(s_{2},(0.5,0.4),(0.5,0.4)\right)\right\}$ \\
\hline
\end{tabular}

$0.5273, G_{5}=0.5332$. Then we can rank the relative closeness coefficients as $G_{1}>G_{2}>G_{5}>G_{4}>G_{3}$. Alternatives can be ranked accordingly as $A_{1} \succ A_{2} \succ$ $A_{5} \succ A_{4} \succ A_{3}$ and the optimal alternative is $A_{1}$. If other cross-entropy measures are used, we can calculate similarly and results are shown in Table 4, where $p=q=2$. For attribute weights $w_{2}$ and $w_{3}$, we can get results as in Table 5 and Table 6 , respectively.

In Table 4 , it can be seen that $A_{2}$ becomes the optimal alternative if cross-entropy measures $C E_{9}^{*}$ and $C E_{10}^{*}$ are used, which is quite different from other results. However, in most cases, the ranking is $A_{1} \succ A_{2} \succ A_{5} \succ A_{4} \succ A_{3}$ and $A_{1}$ becomes the best alternative and $A_{3}$ becomes the worst alternative. The subtle ranking differences are due to the different information fusion mechanisms. In Table 5, $A_{1}$ becomes the optimal alternative if $C E_{1}^{*}, C E_{2}^{*}$,
$C E_{4}^{*}, C E_{7}^{*}$ are used and $A_{2}$ becomes the best alternative for other cross-entropy measures. $A_{3}$ is still the worst alternative. In Table 6, we can get the same ranking $A_{1} \succ A_{2} \succ A_{5} \succ A_{4} \succ A_{3}$ for all the crossentropy measures.

Since different cross-entropy measures may produce different ranking results and each crossentropy has its own characteristics and emphasis, different cross-entropy measures can provide different views of the decision problem. Decision makers can choose the corresponding cross-entropy measure according to real needs, decision makers' preference and interests.

In order to illustrate practical advantages of the new method, we compare it with the method of Peng et al. ${ }^{26}$. In Peng et al.'s method, only hesitant intuitionistic fuzzy values are considered. If linguistic terms are omitted, $C E_{i}^{*}(i=1,2, \ldots, 10)$ reduce to 
Table 3: The results of different aggregation operators with different attribute weights.

\begin{tabular}{|c|c|c|c|c|c|c|c|}
\hline & & $S\left(\breve{h}_{1}\right)$ & $S\left(\breve{h}_{2}\right)$ & $S\left(\breve{h}_{3}\right)$ & $S\left(\breve{h}_{4}\right)$ & $S\left(\breve{h}_{5}\right)$ & Rankings \\
\hline \multirow[t]{3}{*}{$w_{1}$} & LHIFWA & 0.2527 & 0.2493 & 0.2193 & 0.2505 & 0.2126 & $A_{1} \succ A_{4} \succ A_{2} \succ A_{3} \succ A_{5}$ \\
\hline & LHIFWG & 0.2269 & 0.2285 & 0.1901 & 0.2193 & 0.1884 & $A_{2} \succ A_{1} \succ A_{4} \succ A_{3} \succ A_{5}$ \\
\hline & GLHIFWA $_{2}$ & 0.2709 & 0.2626 & 0.2445 & 0.2724 & 0.2283 & $A_{4} \succ A_{1} \succ A_{2} \succ A_{3} \succ A_{5}$ \\
\hline \multirow[t]{3}{*}{$w_{2}$} & LHIFWA & 0.2258 & 0.2599 & 0.2018 & 0.2278 & 0.2057 & $A_{2} \succ A_{4} \succ A_{1} \succ A_{5} \succ A_{3}$ \\
\hline & LHI & 1949 & 0.2297 & 0.1818 & 0.1922 & 0.1838 & \\
\hline & GLHI & 0.2500 & 0.2791 & 0.2212 & 0.2525 & 0.2207 & $A_{2} \succ A_{5} \succ A_{4} \succ A_{1} \succ A_{3}$ \\
\hline \multirow[t]{3}{*}{$w_{3}$} & LHIF & 0.2630 & 0.2215 & 0.1876 & 0.1887 & 0.2442 & $A_{1} \succ A_{5} \succ A_{2} \succ A_{4} \succ A_{3}$ \\
\hline & LHIFWG & 0.2390 & 0.1987 & 0.1654 & 0.1614 & 0.2201 & $A_{1} \succ A_{5} \succ A_{2} \succ A_{3} \succ A_{4}$ \\
\hline & GLHIFWA $_{2}$ & 0.2787 & 0.2363 & 0.2100 & 0.2118 & 0.2582 & $A_{1} \succ A_{5} \succ A_{2} \succ A_{4} \succ A_{3}$ \\
\hline
\end{tabular}

Table 4: The results with known attribute weight vector $w_{1}$.

\begin{tabular}{lccccccc}
\hline & $G_{1}$ & $G_{2}$ & $G_{3}$ & $G_{4}$ & $G_{5}$ & Rankings & Best alternative \\
\hline$C E_{1}^{*}$ & 0.5588 & 0.5414 & 0.4701 & 0.5273 & 0.5332 & $A_{1} \succ A_{2} \succ A_{5} \succ A_{4} \succ A_{3}$ & $A_{1}$ \\
$C E_{2}^{*}$ & 0.6048 & 0.5943 & 0.4865 & 0.5395 & 0.5698 & $A_{1} \succ A_{2} \succ A_{5} \succ A_{4} \succ A_{3}$ & $A_{1}$ \\
$C E_{3}^{*}$ & 0.5410 & 0.5349 & 0.4696 & 0.5133 & 0.5241 & $A_{1} \succ A_{2} \succ A_{5} \succ A_{4} \succ A_{3}$ & $A_{1}$ \\
$C E_{4}^{*}$ & 0.5808 & 0.5670 & 0.4740 & 0.5465 & 0.5439 & $A_{1} \succ A_{2} \succ A_{4} \succ A_{5} \succ A_{3}$ & $A_{1}$ \\
$C E_{5}^{*}$ & 0.5321 & 0.5295 & 0.4633 & 0.4990 & 0.5162 & $A_{1} \succ A_{2} \succ A_{5} \succ A_{4} \succ A_{3}$ & $A_{1}$ \\
$C E_{6}^{*}$ & 0.5264 & 0.5213 & 0.4515 & 0.4913 & 0.5114 & $A_{1} \succ A_{2} \succ A_{5} \succ A_{4} \succ A_{3}$ & $A_{1}$ \\
$C E_{7}^{*}$ & 0.6046 & 0.5895 & 0.4876 & 0.5670 & 0.5751 & $A_{1} \succ A_{2} \succ A_{5} \succ A_{4} \succ A_{3}$ & $A_{1}$ \\
$C E_{8}^{*}$ & 0.5512 & 0.5470 & 0.4763 & 0.5153 & 0.5341 & $A_{1} \succ A_{2} \succ A_{5} \succ A_{4} \succ A_{3}$ & $A_{1}$ \\
$C E_{9}^{*}$ & 0.4120 & 0.4171 & 0.3913 & 0.4009 & 0.3940 & $A_{2} \succ A_{1} \succ A_{4} \succ A_{5} \succ A_{3}$ & $A_{2}$ \\
$C E_{10}^{*}$ & 0.3854 & 0.3874 & 0.3713 & 0.3676 & 0.3644 & $A_{2} \succ A_{1} \succ A_{3} \succ A_{4} \succ A_{5}$ & $A_{2}$ \\
\hline
\end{tabular}

intuitionistic hesitant fuzzy cross-entropy measures $C E_{i}^{\dagger}(i=1,2, \ldots, 10)$. Extend hesitant intuitionistic fuzzy evaluation elements according to the risk attitude of decision makers. Assume decision makers are risk-averse and the minimum intuitionistic fuzzy value is added until all the hesitant intuitionisic fuzzy elements have the same number of intuitionisic fuzzy values and the extended decision matrix $D=\left(h_{i j}^{\prime}\right)_{5 \times 4}$ is formed. Determine the hesitant intuitionistic fuzzy positive ideal solution $h^{+}$and the hesitant intuitionistic fuzzy negative ideal solution $h^{-}$as $h^{+}=\left\{a^{+}\right\}, a^{+}=((1,0),(1,0)), h^{-}=$ $\left\{a^{-}\right\}, a^{-}=((0,1),(0,1))$. Calculate the symmetric cross-entropy measures of $h_{i j}^{\prime}$ from $h^{+}$and $h^{-}$ as $G_{i j}^{+}, G_{i j}^{-}$, respectively. Assume weight vector of attributes is also $w_{1}=(0.15,0.20,0.30,0.35)$ to facilitate comparison. We can calculate weighted relative closeness coefficients of alternatives by using Eq.(38) and results are shown in Table 7. From the results we can see different ranking results can be got in the proposed method and Peng et al. ${ }^{26}$ method. In the proposed method, $A_{1}$ is the optimal alternative in most case and $A_{2}$ is optimal alternative in $C E_{9}^{*}$ and $C E_{10}^{*}$. In Peng et al. ${ }^{26}$ method, $A_{2}, A_{3}$ and $A_{4}$ become the optimal alternative for different cross-entropy measures. Different ranking results due to different decision information. Comparing evaluation information in Peng el al.'s method with that in the proposed algorithm, linguistic terms have been omitted in Peng et al.'s method. If all decision makers use the same linguistic term in evaluation in the proposed method, we can got the same ranking results in the two methods. Since different linguistic terms can be used in the proposed method, more information has been used and more accurate evaluation values can be got. Different evaluation information has been used in two methods and different results are reasonable. We further compare it with the method of Yang et al. ${ }^{54}$. In Yang et al.'s method, each linguistic term only has one intuitionistic fuzzy membership. Hence we first aggregate the intuition- 
Table 5: The results with completely unknown attribute weight vector $w_{2}$.

\begin{tabular}{lccccccc}
\hline & $G_{1}$ & $G_{2}$ & $G_{3}$ & $G_{4}$ & $G_{5}$ & Rankings & Best alternative \\
\hline$C E_{1}^{*}$ & 0.5572 & 0.5480 & 0.4558 & 0.4877 & 0.5339 & $A_{1} \succ A_{2} \succ A_{5} \succ A_{4} \succ A_{3}$ & $A_{1}$ \\
$C E_{2}^{*}$ & 0.4756 & 0.4613 & 0.3137 & 0.3603 & 0.4364 & $A_{1} \succ A_{2} \succ A_{5} \succ A_{4} \succ A_{3}$ & $A_{1}$ \\
$C E_{3}^{*}$ & 0.5392 & 0.5400 & 0.4575 & 0.4828 & 0.5239 & $A_{2} \succ A_{1} \succ A_{5} \succ A_{4} \succ A_{3}$ & $A_{2}$ \\
$C E_{4}^{*}$ & 0.5819 & 0.5689 & 0.4566 & 0.5012 & 0.5419 & $A_{1} \succ A_{2} \succ A_{5} \succ A_{4} \succ A_{3}$ & $A_{1}$ \\
$C E_{5}^{*}$ & 0.5267 & 0.5339 & 0.4492 & 0.4656 & 0.5149 & $A_{2} \succ A_{1} \succ A_{5} \succ A_{4} \succ A_{3}$ & $A_{2}$ \\
$C E_{6}^{*}$ & 0.5442 & 0.5497 & 0.4629 & 0.4824 & 0.5321 & $A_{2} \succ A_{1} \succ A_{5} \succ A_{4} \succ A_{3}$ & $A_{2}$ \\
$C E_{7}^{*}$ & 0.6078 & 0.5929 & 0.4696 & 0.5169 & 0.5798 & $A_{1} \succ A_{2} \succ A_{5} \succ A_{4} \succ A_{3}$ & $A_{1}$ \\
$C E_{8}^{*}$ & 0.5463 & 0.5520 & 0.4615 & 0.4796 & 0.5327 & $A_{2} \succ A_{1} \succ A_{5} \succ A_{4} \succ A_{3}$ & $A_{2}$ \\
$C E_{9}^{*}$ & 0.3955 & 0.4178 & 0.3797 & 0.3946 & 0.3889 & $A_{2} \succ A_{1} \succ A_{4} \succ A_{5} \succ A_{3}$ & $A_{2}$ \\
$C E_{10}^{*}$ & 0.3727 & 0.3902 & 0.3531 & 0.3576 & 0.3586 & $A_{2} \succ A_{1} \succ A_{5} \succ A_{4} \succ A_{3}$ & $A_{2}$ \\
\hline
\end{tabular}

Table 6: The results with partly known attribute weight vector $w_{3}$.

\begin{tabular}{cccccccc}
\hline & $G_{1}$ & $G_{2}$ & $G_{3}$ & $G_{4}$ & $G_{5}$ & Rankings & Best alternative \\
\hline$C E_{1}^{*}$ & 0.6049 & 0.5051 & 0.4501 & 0.4769 & 0.5807 & $A_{1} \succ A_{5} \succ A_{2} \succ A_{4} \succ A_{3}$ & $A_{1}$ \\
$C E_{2}^{*}$ & 0.5457 & 0.3975 & 0.3085 & 0.3498 & 0.5026 & $A_{1} \succ A_{5} \succ A_{2} \succ A_{4} \succ A_{3}$ & $A_{1}$ \\
$C E_{3}^{*}$ & 0.5764 & 0.5076 & 0.4501 & 0.4742 & 0.5601 & $A_{1} \succ A_{5} \succ A_{2} \succ A_{4} \succ A_{3}$ & $A_{1}$ \\
$C E_{4}^{*}$ & 0.6360 & 0.5213 & 0.4482 & 0.4876 & 0.6005 & $A_{1} \succ A_{5} \succ A_{2} \succ A_{4} \succ A_{3}$ & $A_{1}$ \\
$C E_{5}^{*}$ & 0.5691 & 0.5010 & 0.4418 & 0.4546 & 0.5533 & $A_{1} \succ A_{5} \succ A_{2} \succ A_{4} \succ A_{3}$ & $A_{1}$ \\
$C E_{6}^{*}$ & 0.5843 & 0.5139 & 0.4536 & 0.4703 & 0.5686 & $A_{1} \succ A_{5} \succ A_{2} \succ A_{4} \succ A_{3}$ & $A_{1}$ \\
$C E_{7}^{*}$ & 0.6660 & 0.5408 & 0.4617 & 0.5033 & 0.6380 & $A_{1} \succ A_{5} \succ A_{2} \succ A_{4} \succ A_{3}$ & $A_{1}$ \\
$C E_{8}^{*}$ & 0.5914 & 0.5160 & 0.4536 & 0.4684 & 0.5741 & $A_{1} \succ A_{5} \succ A_{2} \succ A_{4} \succ A_{3}$ & $A_{1}$ \\
$C E_{9}^{*}$ & 0.4158 & 0.4029 & 0.3623 & 0.3789 & 0.4099 & $A_{1} \succ A_{5} \succ A_{2} \succ A_{4} \succ A_{3}$ & $A_{1}$ \\
$C E_{10}^{*}$ & 0.3939 & 0.3774 & 0.3374 & 0.3425 & 0.3801 & $A_{1} \succ A_{5} \succ A_{2} \succ A_{4} \succ A_{3}$ & $A_{1}$ \\
\hline
\end{tabular}

istic fuzzy memberships into a collective one by using intuitionistic fuzzy averaging operator (IFA) if a linguistic term has several intuitionistic fuzzy memberships. $\operatorname{IFA}\left(\alpha_{1}, \alpha_{2}, \ldots, \alpha_{n}\right)=\sum_{j=1}^{n} \frac{1}{n} \alpha_{j}=(1-$ $\left.\prod_{j=1}^{n}\left(1-\mu_{j}^{(k)}\right)^{\frac{1}{n}}, \prod_{j=1}^{n}\left(v_{j}\right)^{\frac{1}{n}}\right), \alpha_{j}=\left(\mu_{j}, v_{j}\right)$. The decision matrix degenerates to $\widehat{D}=\left(\widehat{h}_{i j}\right)_{5 \times 4}$. Then we aggregate the evaluation values by using the hesitant intuitionistic fuzzy linguistic weighted averaging (HIFLWA) operator, HIFLWA $\left.\widehat{H}_{w}, \widehat{h}_{2}, \ldots, \widehat{h}_{n}\right)=$ $\sum_{j=1}^{n} w_{j} \widehat{h}_{j}=\bigcup_{a_{i} \in \widehat{h}_{i}}\left\{\left(s_{\left(\sum_{j=1}^{n} w_{j} \theta_{j}\right)},\left(1-\prod_{j=1}^{n}(1-\right.\right.\right.$ $\left.\left.\left.\left.\mu_{j}^{(k)}\right)^{w_{j}}, \prod_{j=1}^{n}\left(v_{j}^{(k)}\right)^{w_{j}}\right)\right)\right\}$, the hesitant intuitionistic fuzzy linguistic weighted geometric (HIFLWG) operator HIFLWG $\left(\widehat{h}_{1}, \widehat{h}_{2}, \ldots, \widehat{h}_{n}\right)=\prod_{j=1}^{n}\left(\widehat{h}_{j}\right)^{w_{j}}=$ $\bigcup_{a_{i} \in \widehat{h}_{i}}\left\{\left(s_{\left(\prod_{j=1}^{n}\left(\theta_{j}\right)^{w_{j}}\right)},\left(\prod_{j=1}^{n}\left(\mu_{j}^{(k)}\right)^{w_{j}}, 1-\prod_{j=1}^{n}(1-\right.\right.\right.$ $\left.\left.\left.\left.v_{j}^{(k)}\right)^{w_{j}}\right)\right)\right\}$, or the generalized hesitant intuitionistic fuzzy linguistic weighted averaging (GHI-
FLWA) operator GHIFLWA $\left(\widehat{h}_{1}, \widehat{h}_{2}, \ldots, \widehat{h}_{n}\right)=$ $\left(\sum_{j=1}^{n} w_{j}\left(\widehat{h}_{j}\right)^{\lambda}\right)^{1 / \lambda}=\bigcup_{a_{i} \in \widehat{h}_{i}}\left\{\left(s_{\left(\sum_{j=1}^{n} w_{j}\left(\theta_{j}\right)^{\lambda}\right)^{1 / \lambda},((1-}\right.\right.$ $\left.\prod_{j=1}^{n}\left(1-\left(\mu_{j}^{(k)}\right)^{\lambda}\right)^{w_{j}}\right)^{1 / \lambda}, 1-\left(1-\prod_{j=1}^{n}(1-(1-\right.$ $\left.\left.\left.\left.\left.\left.v_{j}^{(k)}\right)^{\lambda}\right)^{w_{j}}\right)^{1 / \lambda}\right)\right)\right\}$. The weight vector of attributes is also taken as $w_{1}=(0.15,0.20,0.30,0.35)$ to facilitate comparison. The results are shown in Table 8. From the results we can see that we can get similar ranking results in the proposed method and Yang et al.'s method ${ }^{54}$. There is little difference in values to be aggregated since different intuitionistic fuzzy values in the proposed method are replaced by the average one. For space limit, we only present a simple example to illustrate the new algorithm. The results for other large-scale complex decision problems may have a greater difference. Since different intuitionistic fuzzy values can be used to model hesitation and uncertainty, the proposed method is more flexible and accurate. 
Table 7: The results of Peng et al. ${ }^{29}$ method with known attribute weights $w_{1}$.

\begin{tabular}{cccccccc}
\hline & $G_{1}$ & $G_{2}$ & $G_{3}$ & $G_{4}$ & $G_{5}$ & Rankings & Best alternative \\
\hline$C E_{1}^{\dagger}$ & 0.6797 & 0.6785 & 0.6763 & 0.6955 & 0.6685 & $A_{4} \succ A_{1} \succ A_{2} \succ A_{3} \succ A_{4}$ & $A_{4}$ \\
$C E_{2}^{\dagger}$ & 0.6654 & 0.6799 & 0.6579 & 0.6803 & 0.6496 & $A_{4} \succ A_{2} \succ A_{1} \succ A_{3} \succ A_{5}$ & $A_{4}$ \\
$C E_{3}^{\dagger}$ & 0.6079 & 0.6222 & 0.6008 & 0.6196 & 0.6009 & $A_{2} \succ A_{4} \succ A_{1} \succ A_{5} \succ A_{3}$ & $A_{2}$ \\
$C E_{4}^{\dagger}$ & 0.6636 & 0.6551 & 0.6660 & 0.6637 & 0.6111 & $A_{3} \succ A_{4} \succ A_{1} \succ A_{2} \succ A_{5}$ & $A_{3}$ \\
$C E_{5}^{\dagger}$ & 0.5600 & 0.5732 & 0.5541 & 0.5719 & 0.5538 & $A_{2} \succ A_{4} \succ A_{1} \succ A_{3} \succ A_{5}$ & $A_{2}$ \\
$C E_{6}^{\dagger}$ & 0.6068 & 0.6204 & 0.6007 & 0.6180 & 0.6000 & $A_{2} \succ A_{4} \succ A_{1} \succ A_{3} \succ A_{5}$ & $A_{2}$ \\
$C E_{7}^{\dagger}$ & 0.7206 & 0.7334 & 0.7517 & 0.7276 & 0.7376 & $A_{3} \succ A_{5} \succ A_{2} \succ A_{4} \succ A_{1}$ & $A_{3}$ \\
$C E_{8}^{\dagger}$ & 0.6043 & 0.6272 & 0.6196 & 0.6271 & 0.6128 & $A_{2} \succ A_{4} \succ A_{3} \succ A_{5} \succ A_{1}$ & $A_{2}$ \\
$C E_{9}^{\dagger}$ & 0.7280 & 0.7380 & 0.7284 & 0.7455 & 0.7205 & $A_{4} \succ A_{2} \succ A_{3} \succ A_{1} \succ A_{5}$ & $A_{4}$ \\
$C E_{10}^{\dagger}$ & 0.7046 & 0.7242 & 0.6949 & 0.7221 & 0.6922 & $A_{2} \succ A_{4} \succ A_{1} \succ A_{3} \succ A_{5}$ & $A_{2}$ \\
\hline
\end{tabular}

Table 8: The results of Yang et al. ${ }^{68}$ with weights $w_{1}$.

\begin{tabular}{lllllll}
\hline & $S\left(\widehat{h}_{1}\right)$ & $S\left(\widehat{h}_{2}\right)$ & $S\left(\widehat{h}_{3}\right)$ & $S\left(\widehat{h}_{4}\right)$ & $S\left(\widehat{h}_{5}\right)$ & Rankings \\
\hline HIFLWA & 0.2528 & 0.2499 & 0.2208 & 0.2420 & 0.2128 & $A_{1} \succ A_{2} \succ A_{4} \succ A_{3} \succ A_{5}$ \\
HIFLWG & 0.2275 & 0.2302 & 0.1936 & 0.2021 & 0.1890 & $A_{2} \succ A_{1} \succ A_{4} \succ A_{3} \succ A_{5}$ \\
GHIFLWA $_{2}$ & 0.2709 & 0.2628 & 0.2454 & 0.2650 & 0.2282 & $A_{1} \succ A_{4} \succ A_{2} \succ A_{3} \succ A_{5}$ \\
\hline
\end{tabular}

From the above analysis we can see the proposed approaches have the following advantages. First, LHIFEs have been used to evaluate alternatives, which are more flexible since each LHIFE has several linguistic evaluation values and each linguistic evaluation value has several intuitionistic fuzzy memberships. The inherent fuzzy thought of the decision makers have been retained, which can guarantee accuracy of final results. Second, the crossentropy measures are very important in decision making and we have found few study based on the linguistic hesitant intuitionistic fuzzy information. The new proposed cross-entropy measures can include the advantages of intuitionistic fuzzy crossentropy measures and hesitant fuzzy cross-entropy measures. Finally, the proposed approaches can provide useful and flexible way to deal with multiple attribute decision making problem with different attribute weight situations including attribute weights partly known, completely known and completely unknown.

\section{Conclusions}

In this paper, some linguistic hesitant intuitionistic fuzzy cross-entropy measures have been pro- posed, which have the advantages of the intuitionistic fuzzy cross-entropy measures and hesitant fuzzy cross-entropy measures. We first introduce some aggregation operators including LHIFWA operator, LHIFWG operator and the GLHIFWA operator. Then we propose several linguistic hesitant intuitionistic fuzzy cross-entropy measures. The properties of new cross-entropy measures have been studied. Two new multiple attribute decision making methods have been proposed based on the proposed cross-entropy measures, in which attribute values are given as linguistic hesitant intuitionistic fuzzy elements. The supplier selection problem has been presented to illustrate feasibility and practical advantages of the new methods. The prominent feature of the new methods is that they can provide a flexible and useful way to deal with decision making problems within linguistic hesitant intuitionisic fuzzy environment.

Further improvements of our algorithm might include the application of our new method to more complex multiple attribute decision making problem in reality, such as the personnel selection, the product selection, and the environment evaluation, etc. 


\section{Acknowledgments}

The authors deeply thank anonymous referees and the associate editor for their helpful remarks, which have helped us to improve the paper in both content and style. This work is partly supported by National Natural Science Foundation of China (No. 11401457, 61403298), Postdoctoral Science Foundation of China (2015M582624).

\section{References}

1. Atanassov KT. Intuitionistic fuzzy sets. Fuzzy Sets and Systems 1986; 20(1): 87-96.

2. Torra V. Hesitant fuzzy sets. International Journal of Intelligent Systems 2010; 25(6): 529-539.

3. Torra V, Narukawa Y. On hesitant fuzzy sets and decision, in: The 18th IEEE International Conference on Fuzzy Systems, Jeju Island, Korea, 1378-1382 (2009).

4. Herrera F, Martínez L. An approach for combining linguistic and numerical information based on 2-tuple fuzzy linguistic representation model in decisionmaking. International Journal of Uncertainty Fuzziness and Knowledge-Based Systems 2000; 8: 539562.

5. Dong YC, Xu YF, Yu S. Computing the Numerical Scale of the Linguistic Term Set for the 2-Tuple Fuzzy Linguistic Representation Model. IEEE Transactions on Fuzzy Systems 2009; 17(6): 1366-1378.

6. Pang Q. Wang H. Xu ZS. Probabilistic Linguistic Term Sets in Multi-Attribute Group Decision Making. Information Sciences (in press).

7. Riera JV, Massanet S, Herrera-Viedma E, Torrens J. Some interesting properties of the fuzzy linguistic model based on discrete fuzzy numbers to manage hesitant fuzzy linguistic information. Applied Soft Computing 2015; 36: 383-391.

8. Rodríguez RM, Martínez L. An Analysis of Symbolic Linguistic Computing Models in Decision Makin. International Journal of General Systems 2013; 42(1): 121-136.

9. Rodríguez RM, Bedregal B, Bustince H, Dong YC, Farhadinia B, Kahraman C, Martínez L, Torra V, Xu YJ, Xu ZS, Herrera F. A position and perspective analysis of hesitant fuzzy sets on information fusion in decision making.Towards high quality progress. Information Fusion 2016; 29: 89-97.

10. Rodríguez RM, Martínez L, Torra V, Xu ZS. Herrera F, Hesitant fuzzy sets: State of the art and future directions. International Journal of Intelligent Systems 2014; 29(6): 495-524.
11. Wu ZB, Xu JP. Possibility Distribution-Based Approach for MAGDM With Hesitant Fuzzy Linguistic Information. IEEE Transactions on Cybernetics 2016; 46(3): 694-705.

12. Bedregal B, Reiser R, Bustince H, Lopez-Molina C, Torra V. Aggregation functions for typical hesitant fuzzy elements and the action of automorphisms. Information Sciences 2014; 255: 82-99.

13. Qian G, Wang H, Feng XQ. Generalized hesitant fuzzy sets and their application in decision support system. Knowledge-Based Systems 2013; 37: 357365.

14. Xia MM, Xu ZS. Hesitant fuzzy information aggregation in decision making. International Journal of Approximate Reasoning 2011; 52: 395-407.

15. Wei GW. Hesitant fuzzy prioritized operators and their application to multiple attribute decision making. Knowledge-Based Systems 2012; 31: 176-182.

16. Xu ZS, Xia MM. Distance and similarity measures for hesitant fuzzy sets. Information Sciences 2011; 181: 2128-2138.

17. Zhang ZM. Hesitant fuzzy power aggregation operators and their application to multiple attribute group decision making. Information Sciences 2013; 234: 150-181.

18. Zhu B, Xu ZS, Xia MM. Hesitant fuzzy geometric Bonferroni means. Information Sciences 2012; 205: $72-85$.

19. Xu ZS, Zhang XL. Hesitant fuzzy multi-attribute decision making based on TOPSIS with incomplete weight information. Knowledge-Based Systems 2013; 52: 53-64

20. Zhang N, Wei GW. Extension of VIKOR method for decision making problem based on hesitant fuzzy set. Applied Mathematical Modelling 2013; 37: 49384947.

21. Zhang XL, Xu ZS. The TODIM analysis approach based on novel measured functions under hesitant fuzzy environment. Knowledge-Based Systems 2014; 61: 48-58.

22. Chen N, Xu ZS, Xia MM. Correlation coefficients of hesitant fuzzy sets and their applications to clustering analysis. Applied Mathematical Modelling; 37: 21972211.

23. Farhadinia B. Information measures for hesitant fuzzy sets and interval-valued hesitant fuzzy sets. Information Sciences 2013; 240: 129-144.

24. Xu ZS, Xia MM. On distance and correlation measures of hesitant fuzzy information. International Journal of Intelligent Systems 2011; 26: 410-425.

25. Peng DH, Gao CY, Gao ZF. Generalized hesitant fuzzy synergetic weighted distance measures and their application to multiple criteria decision-making. Applied Mathematical Modelling 2013; 37: 5837-5850.

26. Peng JJ, Wang JQ, Wu XH, Zhang HY, Chen XH. The 
fuzzy cross-entropy for intuitionistic hesitant fuzzy sets and their application in multi-criteria decisionmaking. International Journal of Systems Science 2014; Doi.10.1080/00207721.2014.993744.

27. Chen N, Xu ZS, Xia MM. Interval-valued hesitant preference relations and their applications to group decision making. Knowledge-Based Systems 2013; 37(1): 528-540.

28. Zhao XF, Lin R, Wei GW. Hesitant triangular fuzzy information aggregation based on Einstein operations and their application to multiple attribute decision making. Expert Systems with Applications 2014; 41: 1086-1094.

29. Chen SM, Hong JA. Multicriteria linguistic decision making based on hesitant fuzzy linguistic term sets and the aggregation of fuzzy sets. Information Sciences 2014; 286: 63-74.

30. Liu HB, Rodríguez RM. A fuzzy envelope for hesitant fuzzy linguistic term set and its application to multicriteria decision making. Information Sciences 2014; 258: 220-238.

31. Zhang ZM, Wu C. Hesitant fuzzy linguistic aggregation operators and their applications to multiple attribute group decision making. Journal of Intelligent \& Fuzzy Systems 2014; 26: 2185-2202.

32. Rodríguez RM, Martínez L, Herrera F. Hesitant Fuzzy Linguistic Term Sets for Decision Making. IEEE Transactions on Fuzzy Systems 2012; 20(1): 109-119.

33. Rodríguez RM, Martínez L, Herrera F. A group decision making model dealing with comparative linguistic expressions based on hesitant fuzzy linguistic term sets. Information Sciences 2013; 241: 28-42.

34. Wang H, Xu ZS. Some consistency measures of extended hesitant fuzzy linguistic preference relations. Information Sciences 2015; 297: 316-331.

35. Wang H. Extended hesitant fuzzy linguistic term sets and their aggregation in group decision making. International Journal of Computational Intelligence Systems 2015; 8(1): 14-33.

36. Yang W, Shi JR, Pang YF. Generalized Linguistic Hesitant Intuitionistic Fuzzy Hybrid Aggregation Operators. Mathematical Problems in Engineering 2015; Doi.org/10.1155/2015/983628.

37. Boer P, Kroese D, Mannor S, Rubinstein R. A tutorial on the cross-entropy method. Annals of Operations Research 2005; 134(1): 19-67.

38. Ye J. Multicriteria fuzzy decision-Making method based on the intuitionistic fuzzy cross-entropy. International Conference on Intelligent Human-Machine Systems and Cybernetics, 2009.

39. Kullback S, Leibler A. On the information and sufficiency. Annals and Mathematical Statistics 1951; 22: 79-86.
40. Bhandari D, Pal NR. Some new information measures for fuzzy sets. Information Sciences 1993; 67: 209228.

41. Zhang QS, Jiang SY. A note on information entropy measures for vague sets and its applications. Information Sciences 2008; 178: 4184-4191.

42. Chen XW, Kar S, Ralescu DA. Cross-entropy measure of uncertain variables. Information Sciences 2012; 201: 53-60.

43. Mao JJ, Yao DB, Wang CC. A novel cross-entropy and entropy measures of IFSs and their applications. Knowledge-Based Systems 2013; 48: 37-45.

44. Xia MM, Xu ZS. Entropy/cross entropy-based group decision making under intuitionistic fuzzy environment. Information Fusion 2012; 13: 31-47.

45. Hung WL, Yang MS. On the J-divergence of intuitionistic fuzzy sets with its application to pattern recognition. Information Sciences 2008; 178: 1641-1650.

46. Wang JQ, Li JJ. Multi-criteria fuzzy decision-making method based on cross entropy and score functions. Expert Systems with Applications 2011; 38: 10321038.

47. Qi XW, Liang CY, Zhang JL. Generalized crossentropy based group decision making with unknown expert and attribute weights under interval-valued intuitionistic fuzzy environment. Computer and Industrial Engineering 2015; 79: 52-64.

48. Xu ZS, Xia MM. Hesitant fuzzy entropy and crossentropy and their use in multiattribute decisionmaking. International Journal of Intelligent Systems 2012; 27: 799-822.

49. Vlachos IK, Sergiadis GD. Intuitionistic fuzzy information application to pattern recognition. Pattern Recognition Letters 2007; 28: 197-206.

50. $\mathrm{Xu} \mathrm{ZS}$. Uncertain linguistic aggregation operators based approach to multiple attribute group decision making under uncertain linguistic environment. Information Sciences 2004; 168: 171-184.

51. $\mathrm{Xu} \mathrm{ZS}$. A deviation-based approach to intuitionistic fuzzy multiple attribute group decision making. Group Decision and Negotiation 2010; 19: 57-76.

52. Hwang CL, Yoon K. Multiple Attribute Decision Making Methods and Applications, Springer, Berlin Heidelberg 1981.

53. Chen ZP, Yang W. An MAGDM based on constrained FAHP and FTOPSIS and its application to supplier selection. Applied Mathematical Modelling 2011; 54: 2802-2815.

54. Yang SD, Sun Z, Ju, YB, Qiao CY. A Novel Multiple Attribute Satisfaction Evaluation Approach with Hesitant Intuitionistic Linguistic Fuzzy Information. Mathematical Problems in Engineering 2014; Doi.org/10.1155/2014/692782. 